\title{
The Role of Lossless Systems in Modern Digital Signal Processing: A Tutorial
}

\author{
P. P. VAIDYANATHAN, SENIOR MEMBER, IEEe, AND ZINNUR DOĞANATA, STUdent MEMBER, IEEE
}

\begin{abstract}
Traditionally, lossless network functions and matrices have played an important role in electrical network theory. Many of the basic mathematical concepts and results pertaining to lossless systems, however, continue to have major applications in modern digital signal processing today. This paper is an attempt at a self-contained exposure to discrete-time lossless systems, their properties, and relevance in digital signal processing.
\end{abstract}

\section{INTRODUCTION}

$\mathrm{T}$ RADITIONALLY, lossless network functions and matrices have played a crucial role in classical electrical network theory. Lossless electrical networks (i.e., networks made of inductors, capacitors, transformers, and gyrators but no resistive elements) have been extensively studied during this century [1]-[8]. Many of the theoretical results on lossless systems have been applied to the synthesis of electrical filters [1]-[3], [6]-[8] which provide prescribed attentuation characteristics (as functions of frequency). A complete treatment of continuous-time lossless systems can be found in [4].

If we consider lossless systems from an input-output viewpoint, the theoretical properties of these systems can actually be simulated even without the use of electrical elements. It is in fact possible to build discrete-time systems (and digital filters) by using appropriately defined "lossless building blocks" [9]-[11]. Pioneering contributions in this connection can be found in [9], [39], [49]. An independent development of the concept of losslessness in the discrete-time world is possible [49], [11], and results in a number of exciting applications in modern digital signal processing. These include low sensitivity digital filter design [11]-[13], [49], limit-cycle suppression [10], [14] stability test procedures [15], [16], filter tuning [17], multirate filter banks [18], [20] and development of new sampling-theorems [21] to name a few. The purpose of this tutorial is to introduce some of these ideas in a self-contained way. In order to be compatible with the intended applications, all material will be presented in terms of discrete-time systems. As applications of these concepts in digital signal processing are welldocumented, the primary emphasis in Sections II-IV will

Manuscript received September 18. 1988. This work was supported in part by the National Science Foundation Grants DCI 8552579 and MIP 8604456.

The authors are with the Department of Electrical Engineering, California Institute of Technology, Pasadena, CA 91125.

IEEE Log Number 8929058 be on the theoretical structure and properties of discretetime lossless systems. We hope that such an emphasis paves the way for further study of applications, which are covered in Section V (and in greater details in references mentioned in Sections $\mathrm{V}$ and VI).

Section II introduces the basic concept of losslessness. In Section III we study several algebraic properties of lossless systems. An understanding of these properties is crucial in order to exploit the rich usefulness of lossless systems in digital signal processing. Since lossless systems typically have many input and output terminals, a brief review of multiinput multioutput systems [22], [23] is included in Section III. Next, the most general form of a rational lossless transfer matrix is presented in Section IV, along with synthesis procedures for the FIR case. Our attention in Section IV is mostly restricted to FIR systems for two reasons: first, these systems have been explored to a lesser extent in the past. Second, FIR lossless systems have a special role in multirate digital filter banks (Section V). And finally, FIR lossless systems are just fascinating, easier to handle, and fun to play with. Section V outlines some applications of lossless systems in signal processing, with appropriate pointers to the literature. Section VI includes suggestions for further reading.

\section{Notations}

Boldfaced letters such as $\boldsymbol{A}, \boldsymbol{H}(z)$ etc. denote matrices or vectors. The row and column indices of matrices and vectors are always counted from zero. Superscript $T$ (as in $\boldsymbol{A}^{T}$ ) stands for transposition. Superscript dagger (as in $\boldsymbol{A}^{\dagger}$ ) stands for transposition followed by conjugation. Superscript asteriks (as in $\boldsymbol{A}^{*}, a^{*}$ ), denotes complex conjugation. Subscript asteriks (as in $H_{*}(z)$ ) stands for conjugation of coefficients of $H(z)$. For example, if $H(z)=$ $a+b z^{-1}$, then $H_{*}(z)=a^{*}+b^{*} z^{-1}$. The tilde notation is defined as follows: $\tilde{\boldsymbol{H}}(z)=\boldsymbol{H}_{*}^{T}\left(z^{-1}\right)$. It can be verified that $\tilde{\boldsymbol{H}}\left(e^{j \omega}\right)=\boldsymbol{H}^{\dagger}\left(e^{j \omega}\right)$, i.e., on the unit circle of the $z$ plane, tilde and dagger are synonymous. The acronym LTI stands for linear time invariant systems. The energy of a signal $u(n)$ is denoted by $E_{u}$ (and for a vector signal $\boldsymbol{u}(n)$, by $\left.E_{u}\right)$. By definition, the energy $E_{u}$ of a scalar sequence is given by $E_{u}=\Sigma_{n=-\infty}^{\infty}|u(n)|^{2}$. For a vector sequence $\boldsymbol{u}(n)$, the definition is $E_{\boldsymbol{u}}=\sum_{n=-\infty}^{\infty} \boldsymbol{u}^{\dagger}(n)$ $\boldsymbol{u}(n)$. Determinants of matrix functions are denoted by calligraphic letters; thus det $\boldsymbol{H}(z)=\mathfrak{H C}(z)$, det $\boldsymbol{P}(z)=$ $\mathcal{P}(z)$, and $\operatorname{det} \boldsymbol{Q}(z)=\mathcal{Q}(z)$. The notation $\boldsymbol{P}<\boldsymbol{Q}$ where 
$\boldsymbol{P}$ and $\boldsymbol{Q}$ are two Hermitian matrices means that $\boldsymbol{Q}-\boldsymbol{P}$ is positive definite (and $\boldsymbol{P} \leq \boldsymbol{Q}$ means $\boldsymbol{Q}-\boldsymbol{P}$ is positive semidefinite ).

\section{The Losslessness Property}

Simply stated, a discrete-time ${ }^{1}$ lossless system is a device which conserves energy so that the energy $E_{y}$ of the output sequence is equal to the energy $E_{u}$ of the input sequence except for an arbitrary real scale factor $c>0$. Thus,

$$
E_{y}=c E_{u}
$$

where $c$ is independent of $\boldsymbol{u}(n)$. This definition is not restricted to finite-energy inputs; for if $E_{u}$ happens to be infinite (as in $u(n)=\cos \left(\omega_{0} n\right)$ ), then $E_{y}$ is also infinite, so (1) holds anyway.

Of particular interest to us in this article are linear timeinvariant (LTI) systems [24] characterized by rational transfer functions. Perhaps the simplest example of a lossless transfer function (i.e., transfer function of a lossless system) is a delay $H(z)=z^{-L}$ where $L$ is some integer ( $L=0$ not excluded). A more nontrivial example is a stable all-pass transfer function [24] which satisfies

$$
\left|H\left(e^{j \omega}\right)\right|=d \quad \text { for all } \omega .
$$

where $d>0$ is constant. For such a system we have $\left|Y\left(e^{j \omega}\right)\right|=d\left|U\left(e^{j \omega}\right)\right|$ for every (Fourier transformable) input, so that the following relation holds

$$
\begin{gathered}
\frac{1}{2 \pi} \int_{0}^{2 \pi}\left|Y\left(e^{j \omega}\right)\right|^{2} d \omega=\frac{c}{2 \pi} \int_{0}^{2 \pi}\left|U\left(e^{j \omega}\right)\right|^{2} d \omega, \\
c>0 .
\end{gathered}
$$

According to Parseval's relation [24], the integrals in (3) are precisely the energies $E_{y}$ and $E_{u}$, respectively, which shows that an all-pass function is indeed lossless. In fact, one can also work backwards on this kind of reasoning and prove that a lossless function has to be all-pass.

A familiar example [24] of a first order all-pass function is $H(z)=\left(a^{*}+z^{-1}\right) /\left(1+a z^{-1}\right)$. We see that $H\left(e^{j \omega}\right)$ $=e^{-j \omega}\left(a^{*} e^{j \omega}+1\right) /\left(1+a e^{-j \omega}\right)$ whose magnitude is clearly unity. Even though all-pass functions have several applications [30] (some of which we shall outline in Section V), the usefulness of lossless systems is greatly enhanced by extending the definition to multiinput and multioutput (MIMO) systems.

\section{A. MIMO Lossless Systems}

Fig. 1 shows a two-input two-output LTI system. Here the two output sequences $y_{0}(n)$ and $y_{1}(n)$ are related (in the $z$ domain ) to the input sequences $u_{0}(n)$ and $u_{1}(n)$ by

$$
\begin{aligned}
& Y_{0}(z)=H_{00}(z) U_{0}(z)+H_{01}(z) U_{1}(z) \\
& Y_{1}(z)=H_{10}(z) U_{0}(z)+H_{11}(z) U_{1}(z) .
\end{aligned}
$$

\footnotetext{
'For our purposes, a discrete-time system is a device which produces a unique output signal $\boldsymbol{y}(n)$ in response to an input signal $\boldsymbol{u}(n)$ where $-\infty$ $\leq n \leq \infty$.
}

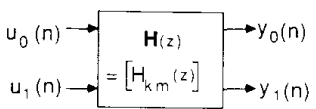

Fig. 1. A two-input two-output LTI system.

The quantity $H_{k m}(z)$ is the transfer function from the $m$ th input to the $k$ th output.

More generally, consider an LTI system with $r$ input sequences $u_{k}(n), 0 \leq k \leq r-1$ and $p$ output sequences $y_{k}(n), 0 \leq k \leq p-1$. (Single-input single-output systems, which have $p=r=1$, are commonly referred to as scalar systems). The $k$ th output sequence $y_{k}(n)$ depends on all the $r$ input sequences so that

$$
Y_{k}(z)=\sum_{m=0}^{r-1} H_{k m}(z) U_{m}(z)
$$

One can neatly summarize these by using matrix notations: define the column vectors

$$
\begin{aligned}
\boldsymbol{u}(n) & =\left[\begin{array}{llll}
u_{0}(n) & u_{1}(n) & \cdots & u_{r-1}(n)
\end{array}\right]^{T}, \\
\boldsymbol{y}(n) & =\left[\begin{array}{llll}
y_{0}(n) & y_{1}(n) & \cdots & y_{p-1}(n)
\end{array}\right]^{T} .
\end{aligned}
$$

Let $\boldsymbol{U}(z)$ be the column vector whose $k$ th component is the $z$ transform of $u_{k}(n)$. Define $\boldsymbol{Y}(z)$ similarly. Then the $r$-input $p$-output LTI system is characterized by the $p \times$ $r$ transfer matrix $\boldsymbol{H}(z)$ which relates $\boldsymbol{Y}(z)$ to $\boldsymbol{U}(z)$ according to

$$
\boldsymbol{Y}(z)=\boldsymbol{H}(z) \boldsymbol{U}(z)
$$

Notice once again that $H_{k m}(z)$ is the transfer function from the $m$ th input to the $k$ th output. We shall restrict our attention only to those systems for which $H_{k m}(z)$ are rational functions, i.e., of the form $N_{k m}(z) / D_{k m}(z)$ where $N_{k m}(z)$ and $D_{k m}(z)$ are finite-degree polynomials in the variable $z$.

It is sometimes helpful to study the LTI system in terms of its unit pulse (or impulse) response [24]. Let $h_{k m}(n)$ be the $k$ th output sequence in response to an impulse applied at the $m$ th input terminal. Then the quantity $H_{k m}(z)$ mentioned earlier is nothing but the $z$ transform of $h_{k m}(n)$. If we define the $p \times r$ matrix $\boldsymbol{h}(n)=\left[h_{k m}(n)\right]$, then $\boldsymbol{H}(z)=\Sigma_{n=-\infty}^{\infty} \boldsymbol{h}(n) z^{-n}$. The matrix $\boldsymbol{h}(n)$ will be called the impulse response matrix of the system. In this paper, we are primarily interested in causal and stable systems. Recall [24] that for such systems $\boldsymbol{h}(n)=\mathbf{0}$ for $n<0$, and moreover the poles of $H_{k m}(z)$ are strictly inside the unit circle. At this point it is worth noting that if the elements $h_{k m}(n)$ are real-valued, then $\boldsymbol{H}(z)$ is real for real values of $z$. Such systems are referred to as "real" systems (and $\boldsymbol{H}(z)$ is then a "real" matrix).

There is a matrix version of Parseval's theorem [24] which gives us the following result:

$$
\frac{1}{2 \pi} \int_{0}^{2 \pi} \boldsymbol{U}^{\dagger}\left(e^{j \omega}\right) \boldsymbol{U}\left(e^{j \omega}\right) d \omega=\sum_{n=-\infty}^{\infty} \boldsymbol{u}^{\dagger}(n) \boldsymbol{u}(n) .
$$


Thus, the left-hand side of $(8)$ is a second way to express the energy $E_{u}$ a vector sequence $\boldsymbol{u}(n)$. For an MIMO lossless system, the energy of the output sequence $y(n)$ is equal to that of the input sequence $\boldsymbol{u}(n)$ (upto a scale factor $c>0$ ). How can we create examples of such systems? The simplest example is of course a system for which $\boldsymbol{H}(z)=\boldsymbol{I}$ (assuming $p=r$ ). A nontrival example is provided by taking $\boldsymbol{H}(z)=\boldsymbol{R}$ where $\boldsymbol{R}$ is a constant unitary matrix, i.e., a matrix $\boldsymbol{R}$ such that

$$
\boldsymbol{R}^{\dagger} \boldsymbol{R}=c_{\boldsymbol{r}}
$$

where $c$ is a positive scalar. (If a unitary matrix is real, it is an orthogonal matrix.) With this choice we have $\boldsymbol{y}(n)$ $=\boldsymbol{R} \boldsymbol{u}(n)$ for all $n$ (this is example of a memoryless system [24] since the output at time $n$ depends only on the input at time $n$ ). As a result

$$
\begin{aligned}
\boldsymbol{y}^{\dagger}(n) \boldsymbol{y}(n) & =\boldsymbol{u}^{\dagger}(n) \boldsymbol{R}^{\dagger} \boldsymbol{R} \boldsymbol{u}(n) \\
& =c \boldsymbol{u}^{\dagger}(n) \boldsymbol{u}(n), \quad c>0 .
\end{aligned}
$$

Summing up for all $n$ it is clear that the energies of the input and output sequences are equal. As a specific example, assume $p=r=2$ and let

$$
\boldsymbol{R}=\left[\begin{array}{rr}
\cos \theta & \sin \theta \\
-\sin \theta & \cos \theta
\end{array}\right], \quad \theta \text { real }
$$

so that (9) holds. This system is shown in Fig. 2(a). Since $\boldsymbol{R}$ is memoryless, the example is trivial in a way. However, as we shall see, the matrix (11) forms the building block for much more complicated lossless systems. In fact, it turns out that any $p \times r$ (FIR or IIR) lossless real transfer matrix can be realized simply by appropriate interconnection of the above $2 \times 2$ building blocks (with delays $z^{-1}$ inserted at appropriate places) as we shall see in Section IV.

It is possible to find matrices $\boldsymbol{R}$ satisfying (9) even if they are not square. For example, with $p=2, r=1$ consider the system

$$
\boldsymbol{R}=\left[\begin{array}{c}
\cos \theta \\
\sin \theta
\end{array}\right]
$$

so that

$$
\boldsymbol{R}^{\dagger} \boldsymbol{R}=\cos ^{2} \theta+\sin ^{2} \theta=1 .
$$

Fig. 2(b) shows this lossless "system,", which merely splits the input signal $u_{0}(n)$ in a neat way, so that the total energy is conserved.

In analogy with the memoryless examples above, we can define a general lossless transfer matrix $\boldsymbol{H}(z)$ to be one for which the frequency response $\boldsymbol{H}\left(e^{j \omega}\right)$ is unitary for all $\boldsymbol{\omega}$. In other words, $\boldsymbol{H}(z)$ is unitary on the unitcircle of the $z$ plane. Since $\boldsymbol{Y}\left(e^{j \omega}\right)=\boldsymbol{H}\left(e^{j \omega}\right) \boldsymbol{U}\left(e^{j \omega}\right)$, unitariness of $\boldsymbol{H}\left(e^{j \omega}\right)$ implies

$$
\begin{aligned}
\boldsymbol{Y}^{\dagger}\left(e^{j \omega}\right) \boldsymbol{Y}\left(e^{j \omega}\right) & =\boldsymbol{U}^{\dagger}\left(e^{j \omega}\right) \boldsymbol{H}^{\dagger}\left(e^{j \omega}\right) \boldsymbol{H}\left(e^{j \omega}\right) \boldsymbol{U}\left(e^{j \omega}\right) \\
& =c \boldsymbol{U}^{\dagger}\left(e^{j \omega}\right) \boldsymbol{U}\left(e^{j \omega}\right),
\end{aligned}
$$

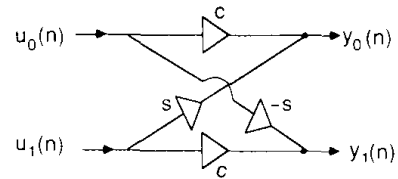

(a)

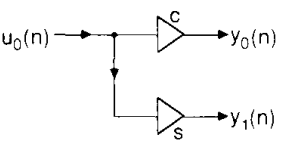

(b)
Fig. 2. Examples of (memoryless) lossless systems. Here $c=\cos \theta$ and $s$ $=\sin \theta$.

with $c>0$. Integrating with respect to $\omega$ in the range 0 $\leq \omega \leq 2 \pi$, and applying Parseval's relation (8), we verify that the energy of the output sequence $y(n)$ is equal to that of the input $\boldsymbol{u}(n)$ (upto a scalar constant). The unitariness of $\boldsymbol{H}(z)$ on the unit circle, viz.,

$$
\boldsymbol{H}^{\dagger}\left(e^{j \omega}\right) \boldsymbol{H}\left(e^{j \omega}\right)=\mathrm{c} \boldsymbol{I}_{r} \text { for all } \omega
$$

can be rewritten using the notations introduced in Section I as

$$
\tilde{\boldsymbol{H}}(z) \boldsymbol{H}(z)=c \boldsymbol{I}_{r},
$$

for $z=e^{j \omega}$. Note that the property (2) satisfied by a scalar allpass function is simply a special case of (15) with $p=$ $r=1$. As a result (16) is sometimes referred to as the allpass property of the transfer matrix $\boldsymbol{H}(z)$. Since (16) holds for every point on the unit circle, and since $\boldsymbol{H}(z)$ and $\tilde{\boldsymbol{H}}(z)$ are analytic (except at an isolated set of points) in the $z$ plane, we conclude that (16) holds for all values of $z$ in the $z$ plane. This follows from a property called analytic continuation [4], [5].

It helps to gain a better understanding of (16) in terms of the columns of $\boldsymbol{H}(z)$. Let $\boldsymbol{H}_{m}(z)$ denote the $m$ th column of $\boldsymbol{H}(z)$. There are $r$ such columns, and each of these is a p-component vector. Condition (16) can be rewritten as

$$
\boldsymbol{H}_{m}^{\dagger}\left(e^{j \omega}\right) \boldsymbol{H}_{n}\left(e^{j \omega}\right)= \begin{cases}c & \text { if } m=n \\ 0 & \text { otherwise. }\end{cases}
$$

In other words, the columns of $\boldsymbol{H}\left(e^{j \omega}\right)$ are mutually orthogonal, and the components $H_{k m}\left(e^{j \omega}\right)$ of the $m$ th column satisfy the property

$$
\sum_{k=0}^{p-1}\left|H_{k m}\left(e^{j \omega}\right)\right|^{2}=c \quad \text { for all } \omega .
$$

A set of $p$ transfer functions $H_{k m}(z), 0 \leq k \leq p-1$ satisfying (18) is called a power complementary set, and (18) is called the power complementary property. In particular, two scalar transfer functions $H(z)$ and $G(z)$ satisfying $\left|G\left(e^{j \omega}\right)\right|^{2}+\left|H\left(e^{j \omega}\right)\right|^{2}=c$ are said to form a power complementary pair.

Property (18) means that each column of $\boldsymbol{H}(z)$ itself represents a lossless system with $p$ outputs and one input. In fact any subset of columns of a lossless $\boldsymbol{H}(z)$ forms a lossless subsystem with fewer than $r$ inputs.

A rectangular $p \times r$ lossless transfer matrix must satisfy $p \geq r$. If $p<r$, then $\boldsymbol{H}^{\dagger}\left(e^{j \omega}\right) \boldsymbol{H}\left(e^{j \omega}\right)$ has rank $\leq p$, so it cannot possibly be equal to $\boldsymbol{I}_{r}$. If a lossless transfer matrix is square (i.e., $p=r$ ) then $\boldsymbol{H}^{T}(z)$ is also lossless. 
This is because, if a square matrix is unitary, then so is its transpose. However, if $p>r$, then $\boldsymbol{H}^{T}(z)$ cannot be lossless.

This seems to be a nice place to write down the definition of a discrete-time lossless system:

Definition 2.1: A $p \times r$ transfer matrix $\boldsymbol{H}(z)$ is said to be lossless if it is stable and is unitary on the unit circle, i.e., satisfies (15) where $c$ is a positive scalar. A lossless $\boldsymbol{H}(\boldsymbol{z})$ is said to be lossless bounded real (abbreviated LBR) if $\boldsymbol{H}(z)$ is real for real $z$.

Definition 2.2: If property (16) holds for all $z$, then $\boldsymbol{H}(z)$ is said to be paraunitary. Thus, a lossless system is stable and paraunitary.

We define a denormalized lossless system to be a stable system satisfying a generalized version of (16), viz., $\tilde{\boldsymbol{H}}(z)$ $\boldsymbol{H}(z)=\boldsymbol{C}$, where $\boldsymbol{C}$ is a positive definite matrix. With $\boldsymbol{C}$ $=c I$ we have the lossless case. Furthermore, if $c=1$, we have the normalized lossless case. Clearly the modified matrix $\boldsymbol{H}(z) \boldsymbol{C}^{1 / 2}$ is lossless in the normalized sense. In this paper we shall consider only the $\boldsymbol{C}=c \boldsymbol{I}$ case.

Comment on Energy Conservation: We know that any $p \times r$ unitary matrix satisfies the property $\boldsymbol{u}^{\dagger} \boldsymbol{R}^{\dagger} \boldsymbol{R} \boldsymbol{u}=c \boldsymbol{u}^{\dagger} \boldsymbol{u}$ for every vector $u$. The converse, which is often not emphasized, is also true, i.e., if a matrix $R$ is such that $\boldsymbol{u}^{\dagger} \boldsymbol{R}^{\dagger} \boldsymbol{R} \boldsymbol{u}=c \boldsymbol{u}^{\dagger} \boldsymbol{u}$ for all vectors $\boldsymbol{u}$ then it is necessarily unitary. In a similar way, if a $p \times r$ transfer matrix $\boldsymbol{H}(z)$ is such that the input sequence and output sequence have same energy (for every possible input sequence) then the system is indeed paraunitary. This converse will not be proved here for sake of brevity.

\section{B. Interconnections of Lossless Systems}

The sum of two lossless functions is in general not lossless. For example, let $H_{0}(z)=1$ and $H_{1}(z)=z^{-1}$ which are both lossless. If we let $H(z)=1+z^{-1}$ then $\left|H\left(e^{j \omega}\right)\right|$ $=2 \cos (\omega / 2)$ which of course is not constant for all $\omega$. The product of two lossless functions (or matrices if product is defined) on the other hand, can be readily verified to be lossless (by using the fact that product of unitary matrices is unitary). This result will be repeatedly used in this article. As a first application, let us construct some examples of non trivial lossless matrices. We know that (11) is lossless; we can verify that the matrix

$$
\mathbf{\Lambda}(z)=\left[\begin{array}{ll}
1 & 0 \\
0 & z^{-1}
\end{array}\right]
$$

is also lossless. If we define

$$
\begin{aligned}
\boldsymbol{G}(z)= & {\left[\begin{array}{rr}
\cos \theta & \sin \theta \\
-\sin \theta & \cos \theta
\end{array}\right]\left[\begin{array}{ll}
1 & 0 \\
0 & z^{-1}
\end{array}\right] } \\
& \cdot\left[\begin{array}{rr}
\cos \alpha & \sin \alpha \\
-\sin \alpha & \cos \alpha
\end{array}\right], \quad \theta, \alpha \text { real }
\end{aligned}
$$

then $\boldsymbol{G}(z)$ is lossless. For example let $\theta=\alpha=\pi / 4$, then

$$
\boldsymbol{G}(z)=\frac{1}{2}\left[\begin{array}{cc}
1-z^{-1} & 1+z^{-1} \\
-\left(1+z^{-1}\right) & -\left(1-z^{-1}\right)
\end{array}\right] .
$$

Notice that (21) represents an FIR system. This example therefore demonstrates a very important point: a MIMO FIR system can be all-pass (i.e., lossless) without being a trivial delay operator! Notice in contrast, that a scalar FIR allpass system is necessarily of the form $z^{-L}$.

Here is an "obvious example" of a MIMO IIR lossless system: $\boldsymbol{H}(z)=A(z) \boldsymbol{I}$ where $\boldsymbol{A}(z)$ is a stable (scalar) allpass function. A second example is a product of the form $\boldsymbol{R} \boldsymbol{\Lambda}$ ( $z$ ) $\boldsymbol{S}$ where $\boldsymbol{R}$ and $\boldsymbol{S}$ are constant unitary matrices and $\boldsymbol{\Lambda}(z)$ is a diagonal matrix of all-pass functions. A more complicated example can be generated by use of feedback connections, as in Fig. 3. Here the vector signals $\boldsymbol{u}_{0}(n), \boldsymbol{u}_{1}(n), \boldsymbol{y}_{0}(n)$ and $\boldsymbol{y}_{1}(n)$ are constrained in the $z$ domain by the relation

$$
\left[\begin{array}{l}
\boldsymbol{Y}_{0}(z) \\
\boldsymbol{Y}_{1}(z)
\end{array}\right]=\left[\begin{array}{ll}
\boldsymbol{T}_{00}(z) & \boldsymbol{T}_{01}(z) \\
\boldsymbol{T}_{10}(z) & \boldsymbol{T}_{11}(z)
\end{array}\right]\left[\begin{array}{c}
U_{0}(z) \\
U_{1}(z)
\end{array}\right]
$$

At the same time the transfer matrix $\boldsymbol{G}(z)$ constrains $\boldsymbol{u}_{1}(n)$ such that

$$
U_{1}(z)=\boldsymbol{G}(z) Y_{1}(z)
$$

Eliminating $U_{1}(z)$ and $Y_{1}(z)$ between (22), (23) we obtain $\boldsymbol{Y}_{0}(z)=\boldsymbol{H}(z) \boldsymbol{U}_{0}(z)$ where

$$
\begin{aligned}
H(z)= & T_{00}(z)+T_{01}(z) G(z) \\
& \cdot\left[I-T_{11}(z) G(z)\right]^{-1} T_{10}(z) .
\end{aligned}
$$

If we now assume that the matrix in (22) composed of the submatrices $\boldsymbol{T}_{k m}(z)$ is lossless with $c=1$, we have the following energy balance equation:

$$
E_{y 0}+E_{y_{1}}=E_{u 0}+E_{u \mid} .
$$

If in addition $G(z)$ is also lossless with $c=1$, we have $E_{y_{l}}=E_{u_{1}}$ which along with (25) implies $E_{y_{0}}=E_{u_{0}}$ ! Thus the matrix $\boldsymbol{H}(z)$ which relates $\boldsymbol{u}_{0}(n)$ to $\boldsymbol{y}_{0}(n)$ conserves energy, so that it is paraunitary. As a specific example let

$$
\boldsymbol{T}(z)=\frac{1}{\sqrt{2}}\left[\begin{array}{ccc}
1 & 1 & 0 \\
\frac{1}{\sqrt{2}} & -\frac{1}{\sqrt{2}} & 1 \\
\frac{1}{\sqrt{2}} & -\frac{1}{\sqrt{2}} & -1
\end{array}\right] .
$$

This matrix is orthogonal, i.e., $\boldsymbol{T}^{T} \boldsymbol{T}=\boldsymbol{I}$, as can be verified explicitly. Let $\boldsymbol{G}(z)=z^{-1}$ which is a scalar lossless function. In this example, we therefore identify $\boldsymbol{T}_{i j}(z)$ as

$$
\boldsymbol{T}_{00}=\frac{1}{\sqrt{2}}\left[\begin{array}{cc}
1 & 1 \\
\frac{1}{\sqrt{2}} & \frac{-1}{\sqrt{2}}
\end{array}\right], \quad \boldsymbol{T}_{01}=\left[\begin{array}{l}
0 \\
\frac{1}{\sqrt{2}}
\end{array}\right]
$$

and

$$
\boldsymbol{T}_{10}=\left[\begin{array}{ll}
\frac{1}{2} & -\frac{1}{2}
\end{array}\right], \quad T_{11}(z)=-\frac{1}{\sqrt{2}} .
$$




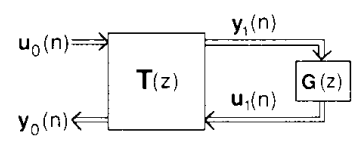

Fig. 3. A feedback connection, giving rise to a lossless IIR system

Using (24) we obtain upon simplification,

$$
\begin{aligned}
\boldsymbol{H}(z)= & \frac{1}{\sqrt{2}}\left[\begin{array}{cc}
1 & 1 \\
\frac{1}{\sqrt{2}} & -\frac{1}{\sqrt{2}}
\end{array}\right]+\frac{z^{-1}}{1+\frac{1}{\sqrt{2}} z^{-1}} \\
& \cdot\left[\begin{array}{cc}
0 & 0 \\
\frac{1}{2 \sqrt{2}} & -\frac{1}{2 \sqrt{2}}
\end{array}\right] .
\end{aligned}
$$

Because of the energy balance proved above, $\boldsymbol{H}(z)$ is paraunitary. Since the pole is at $z=-1 / \sqrt{2}, \boldsymbol{H}(z)$ is stable as well, and hence lossless.

\section{Properties of Lossless LTI Systems}

The unitary property (15) of a stable $\boldsymbol{H}(z)$ induces several other beautiful secondary properties on $\boldsymbol{H}(z)$. Knowledge of these results is crucial for the understanding and design of several modern digital signal processing algorithms. In this section we outline these properties. We begin with a review of standard notions in MIMO system theory.

\section{A. Review of Standard Notions for MIMO LTI Systems}

Recall that a wide class of scalar LTI systems can be described by transfer functions which are ratios of polynomials, i.e., $H(z)=P(z) / Q(z)$ with

$$
P(z)=\sum_{n=0}^{N} p_{n} z^{N-n}, \quad Q(z)=\sum_{n=0}^{N} q_{n} z^{N-n} .
$$

Such transfer functions $H(z)$ are said to be rational. ${ }^{2}$ The expression $P(z) / Q(z)$ is said to be in irreducible form if there are no common factors between $P(z)$ and $Q(z)$. Such a pair of polynomials $P(z), Q(z)$ is said to be relatively prime. With $P(z)$ and $Q(z)$ relatively prime, the zeros of $P(z)$ and $Q(z)$ are respectively the zeros and poles of $H(z)$. A direct-form implementation [24] of an irreducible $P(z) / Q(z)$ requires only $N$ delay elements, which is the smallest possible. The smallest number of delays required to implement $H(z)$ is called the degree of $H(z)$.

An MIMO system characterized by a transfer matrix $\boldsymbol{H}(z)$ can often be given a matrix fraction description (abbreviated MFD). This description is an extension of the rational expression $P(z) / Q(z)$ of the scalar case. The form $\boldsymbol{H}(z)=\boldsymbol{Q}_{l}^{-1}(z) \boldsymbol{P}_{l}(z)$ is called a left MFD, whereas the form $\boldsymbol{H}(z)=\boldsymbol{P}_{r}(z) \boldsymbol{Q}_{r}^{-1}(z)$ is called a right MFD. A transfer matrix can be described by a right MFD if and

\footnotetext{
2Irrational transfer functions [ such as, for example exp $(1 / z)]$ cannot be implemented as finite-order difference equations, and are therefore of little practical interest.
}

only if it can be described by a left MFD [22], [23]. The quantities $\boldsymbol{P}_{l}(z), \boldsymbol{Q}_{l}(z), \boldsymbol{P}_{r}(z)$, and $\boldsymbol{Q}_{r}(z)$ are matrices whose entries are polynomials in $z$. In this paper we shall use only the left MFD description. Deleting the subscript $l$ for simplicity, we then have

$$
\boldsymbol{H}(z)=\boldsymbol{Q}^{-1}(z) \boldsymbol{P}(z)
$$

for the rest of the paper. The matrices $\boldsymbol{Q}(z)$ and $\boldsymbol{P}(z)$ will respectively be called the denominator and numerator of the MFD. For a $r$-input $p$-output system which has a $p \times$ $r$ transfer matrix $\boldsymbol{H}(z)$, the matrix $\boldsymbol{Q}(z)$ is $p \times p$, whereas $\boldsymbol{P}(z)$ is $p \times r$. These polynomial matrices can be expressed as

$$
\boldsymbol{P}(z)=\sum_{n=0}^{N} \boldsymbol{p}_{n} z^{N-n}, \quad \boldsymbol{Q}(z)=\sum_{n=0}^{N} \boldsymbol{q}_{n} z^{N-n}
$$

where $q_{n}$ are $p \times p$ and $p_{n}$ are $p \times r$. Notice that only positive powers of $z$ appear in (32). Readers more familiar with polynomials in $z^{-1}$ can multiply the matrices $Q(z)$ and $\boldsymbol{P}(z)$ with the scalar $z^{-N}$ to obtain equivalent descriptions for $\boldsymbol{H}(z)$.

The smallest number of scalar delay elements $z^{-1}$ required to implement $\boldsymbol{H}(z)$ is called the degree of $\boldsymbol{H}(z)$ (also referred to as the McMillan-degree [23]). In this paper, the degree is always denoted by $K-1$. This is different from $N$ appearing in (32), as can be seen from the example $\boldsymbol{H}(z)=z^{-1} \boldsymbol{I}_{p}$ which is a system with $p=r$, $\boldsymbol{Q}(z)=z \boldsymbol{I}_{p}$ and $\boldsymbol{P}(z)=\boldsymbol{I}_{p}$. This system merely delays each input sequence by one unit. Clearly, $N=1$ here even though we require $p$ delays for the implementation.

More generally, consider the example $\boldsymbol{H}(z)=z^{-1} \boldsymbol{A}$ where $\boldsymbol{A}$ is a constant $p \times r$ matrix with rank $s$. We then have [32] $\boldsymbol{A}=\boldsymbol{B} \boldsymbol{R} \boldsymbol{C}$ where $\boldsymbol{R}$ is $s \times s$ with rank $s$. Thus, $\boldsymbol{H}(z)=\boldsymbol{B}\left[z^{-1} \boldsymbol{R}\right] \boldsymbol{C}$ so that it can be implemented with $s$ delays. So the degree of $z^{-1} \boldsymbol{A}$ is equal to the rank of $\boldsymbol{A}$.

1) Irreducible $M F D$ 's: How does one determine the degree of an arbitrary $\boldsymbol{H}(z)$ ? This turns out to be trickier than appears at first sight. In analogy with the scalar case, we begin by defining an irreducible MFD. First, given the polynomial matrices $\boldsymbol{Q}(z)$ and $\boldsymbol{P}(z)$, if we can write them in the form

$$
\boldsymbol{Q}(z)=L(z) \boldsymbol{Q}_{1}(z), \boldsymbol{P}(z)=L(z) \boldsymbol{P}_{1}(z)
$$

where $L(z), Q_{1}(z)$ and $\boldsymbol{P}_{1}(z)$ are polynomial matrices, then $L(z)$ is said to be a left common divisor (LCD) of $\boldsymbol{Q}(z)$ and $\boldsymbol{P}(z)$. Notice that $\boldsymbol{Q}_{1}^{-1}(z) \boldsymbol{P}_{1}(z)$ is a valid MFD for $\boldsymbol{H}(z)$ because $\boldsymbol{L}(z)$ cancels off in $(31)$.

An $\operatorname{LCD} \boldsymbol{L}(z)$ of $\boldsymbol{Q}(z)$ and $\boldsymbol{P}(z)$ is said to be a greatest LCD (abbreviated GLCD) of $\boldsymbol{Q}(z)$ and $\boldsymbol{P}(z)$ if every other LCD $L_{1}(z)$ of $\boldsymbol{Q}(z)$ and $\boldsymbol{P}(z)$ is a left factor of $\boldsymbol{L}(z)$, i.e.,

$$
L(z)=L_{1}(z) W_{1}(z)
$$

for some polynomial matrix $\boldsymbol{W}_{1}(z)$. An irreducible MFD can be obtained by canceling off a greatest LCD from $\boldsymbol{Q}(z)$ and $\boldsymbol{P}(z)$ in (31). This results in a reduced degree for the denominator determinant, i.e., $\operatorname{deg} \operatorname{det} \boldsymbol{Q}_{1}(z)<$ deg det $\boldsymbol{Q}(z)$, since

$$
Q(z)=\mathscr{L}(z) Q_{1}(z) .
$$


Assuming that we have identified and cancelled off a GLCD, the resulting MFD is said to be irreducible. It can be shown [23] that the degree of $\boldsymbol{H}(z)$ (i.e., the minimum number of scalar delays $z^{-1}$ required to realize it ) is equal to deg det $\boldsymbol{Q}_{1}(z)$ where $\boldsymbol{Q}_{1}(z)$ is the denominator of an irreducible MFD of $\boldsymbol{H}(z)$.

2) Unimodular Matrices: The concept of unimodular matrix polynomials (or simply unimodular matrices [58, vol. 1, ch. VI]) is very important in this context. A matrix polynomial $\boldsymbol{W}(z)$ is said to be unimodular if it is square, and det $W(z)=c \neq 0$ where $c$ is a constant (i.e., independent of $z$ ). A simple example is a triangular matrix with constant elements on the diagonal:

$$
\boldsymbol{W}(z)=\left[\begin{array}{ll}
1 & 0 \\
z & 1
\end{array}\right]
$$

More complicated examples of unimodular matrices, such as

$$
\boldsymbol{W}(z)=\left[\begin{array}{ll}
z^{2}+3 z+3 & z+2 \\
z^{3}+4 z^{2}+8 z+5 & z^{2}+3 z+4
\end{array}\right]
$$

can be generated by multiplying simple unimodular matrices. Given an irreducible MFD for $\boldsymbol{H}(z)$ as in (31), we can find an infinite number of other irreducible MFD's by defining $\boldsymbol{Q}_{1}(z)=\boldsymbol{W}(z) \boldsymbol{Q}(z)$, and $\boldsymbol{P}_{1}(z)=\boldsymbol{W}(z) \boldsymbol{P}(z)$ where $W(z)$ is any $p \times p$ unimodular matrix. The reason is that $Q(z)$ and $Q_{1}(z)$ have the same determinantal degrees. The matrices $Q(z)$ and $P(z)$ describing an irreducible left MFD are said to be left coprime. This means that any GLCD of $\boldsymbol{Q}(z)$ and $\boldsymbol{P}(z)$ is unimodular.

3) State-Space Descriptions: Consider any implementation of the MFD (31). No matter how complicated the implementation is, it is merely an interconnection of scalar delays, scalar multipliers and two-input adders [24] Let $d$ be the number of delays used. Clearly $d \geq K-1$, and the implementation is minimal (or canonic) in delays if $d=K-1$. In any case, assign the names $x_{k}(n)$ to the output sequences of the delay elements, with $0 \leq k \leq d$ -1 . Then the system can be described by the set of statespace equations [25]

$$
\begin{aligned}
\boldsymbol{x}(n+1) & =\boldsymbol{A} \boldsymbol{x}(n)+\boldsymbol{B} \boldsymbol{u}(n), \\
\boldsymbol{y}(n) & =\boldsymbol{C} \boldsymbol{x}(n)+\boldsymbol{D} \boldsymbol{u}(n)
\end{aligned}
$$

where $\boldsymbol{x}(n)=\left[x_{0}(n) x_{1}(n) \cdots x_{d-1}(n)\right]^{T}$ is the state vector. The state-transition matrix $\boldsymbol{A}$ is $d \times d$, and the other matrices have obvious dimensions. The impulse response of the system is given by

$$
\boldsymbol{h}(n)= \begin{cases}0 & n<0 \\ \boldsymbol{D} & n=0 \\ \boldsymbol{C A}^{n-1} \boldsymbol{B} & n>0 .\end{cases}
$$

If an implementation is minimal in delays then the size of $\boldsymbol{A}$ is the smallest possible (viz., $(K-1) \times(K-1)$ ).

The system described by (36), (37) is said to be controllable if we can take it to a prescribed state $x(n)$ at time $n$ by applying a finitely long input sequence $\boldsymbol{u}(m)$, $m_{0} \leq m<n$. The system is said to be observable, if we can compute the state vector $\boldsymbol{x}(n)$ based on finitely many samples of the output sequence, $\boldsymbol{y}(m), n \leq m \leq m_{1}$.

Here are some major results from system-theory: the system (36), (37) is observable if and only if the $p d \times d$ matrix

$$
\boldsymbol{M}_{o}=\left[\begin{array}{c}
\mathrm{C} \\
\boldsymbol{C A} \\
\vdots \\
C A^{d-1}
\end{array}\right]
$$

has full rank, viz, $d$. The system is controllable if and only if the matrix formed by replacing $\boldsymbol{A}$ with $\boldsymbol{A}^{T}$ and $\boldsymbol{C}$ with $\boldsymbol{B}^{T}$ in (39) has full rank $d$. A very interesting way to test observability of a system is provided by the following result [23]: a system is observable if and only if there does not exist an eigenvector $\boldsymbol{v}$ of $\boldsymbol{A}$ such that it is orthogonal to all rows of $\boldsymbol{C}$. Finally, an implementation is minimal in delays if and only if it is both controllable and observable. A minimal-delay implementation is stable if and only if all the eigenvalues $\lambda_{k}$ of $\boldsymbol{A}$ satisfy $\left|\lambda_{k}\right|<1$.

4) Warning About Determinantal Degree of $\boldsymbol{H}(z)$ : Assuming that $\boldsymbol{H}(z)$ is square (i.e., $p=r$ ), we have det $\boldsymbol{H}(z)=\operatorname{det} \boldsymbol{P}(z) / \operatorname{det} \boldsymbol{Q}(z)$. Even if $\boldsymbol{Q}(z)$ and $\boldsymbol{P}(z)$ are left coprime, it is possible for the determinants of $\boldsymbol{Q}(z)$ and $\boldsymbol{P}(z)$ to have common factors! As an example, consider

$$
\begin{aligned}
\boldsymbol{H}(z) & =\left[\begin{array}{ll}
\frac{z}{z+0.5} & 0 \\
0 & \frac{z+0.5}{z}
\end{array}\right] \\
& =\left[\begin{array}{ll}
z+0.5 & 0 \\
0 & z
\end{array}\right]^{-1}\left[\begin{array}{ll}
z & 0 \\
0 & z+0.5
\end{array}\right] .
\end{aligned}
$$

Clearly det $\boldsymbol{Q}(z)=\operatorname{det} \boldsymbol{P}(z)=z(z+0.5)$, so that deg det $\boldsymbol{H}(z)=0$. Thus, the determinantal degree of $\boldsymbol{H}(z)$ does not in general give us an indication of the degree of $\boldsymbol{H}(z)$ (which is clearly greater than 0 here). However, the determinantal degree of $\boldsymbol{Q}(z)$ is equal to the degree of $\boldsymbol{H}(z)$ as long as $\boldsymbol{P}(z)$ and $\boldsymbol{Q}(z)$ are left coprime.

5) Poles and Zeros: The transfer matrix $\boldsymbol{H}(z)$ is said to have a pole at $z_{p}$ if any of its entries $H_{k m}(z)$ has a pole at $z_{p}$. Assuming that the MFD in (31) is irreducible, it turns out that $z_{p}$ is a pole of $\boldsymbol{H}(z)$ if and only if it is a zero of the polynomial det $Q(z)$.

The definition of a zero of the system $\boldsymbol{H}(z)$ is more involved. For this, assuming that $(31)$ is irreducible, we first define the normal rank $\rho_{p}$ of $\boldsymbol{P}(z)$ to be

$$
\max _{z}[\operatorname{rank} P(z)]
$$

Clearly, $\rho_{p} \leq \min (p, r)$. Now, we define $z_{0}$ to be a zero of the system $\boldsymbol{H}(z)$, if the rank of $\boldsymbol{P}\left(z_{0}\right)$ is less than $\rho_{p}$. 
The physical meaning of a zero in terms of the inputoutput behavior is studied in [22]. For a system with $\rho_{p}$ $=p=r$ (which is of considerable interest), the zeros of $\boldsymbol{H}(z)$ coincide with the zeros of $\operatorname{det} \boldsymbol{P}(z)$. Notice, in any case, that this definition reduces to the conventional definition of a zero, when $p=r=1$. Notice also that for any $p \times r$ MFD, the normal rank of $Q(z)$ is equal to $p$ so that the inverse $Q^{-1}(z)$ in (31) is meaningful.

It is possible for a system to have an uncanceled polezero pair at the same point. For example, in (40), there is a pole at $z=-0.5$ and a zero at this same point, which do not cancel.

6) Bezout's Identity: It is a well-known result in classical number theory $[53$, p. 91$]$ that two integers $P$ and $Q$ are relatively prime (i.e., integers with no common integer factors greater than unity), if and only if there exist integers $X, Y$ such that $P X+Q Y=1$. Surprisingly, if $P$ and $Q$ are replaced with polynomials (more generally, matrix polynomials), a similar result holds [22], [23], [55] which says that $\boldsymbol{P}(z)$ and $\boldsymbol{Q}(z)$ in (31) are left coprime (so that the MFD is irreducible) if and only if there exist polynomial and matrices $\boldsymbol{X}(z)$ and $\boldsymbol{Y}(z)$ such that

$$
\boldsymbol{P}(z) \boldsymbol{X}(z)+\boldsymbol{Q}(z) \boldsymbol{Y}(z)=\boldsymbol{I} \text {. }
$$

One consequence of this is that, if $\boldsymbol{P}(z)$ and $\boldsymbol{Q}(z)$ are leftcoprime, there does not exist a vector $v \neq 0$ such that $\boldsymbol{v}^{T} \boldsymbol{P}\left(z_{0}\right)=\mathbf{0}$ and $\boldsymbol{v}^{T} \boldsymbol{Q}\left(z_{0}\right)=\mathbf{0}$ simultaneously for some $z_{0}$. We shall apply this result in the next subsection.

7) FIR Systems and McMillan Degree: Recall that the degree of the system $z^{-1} \boldsymbol{A}$ is equal to the rank of $\boldsymbol{A}$. More generally, consider $\boldsymbol{H}(z)=\Sigma_{n=0}^{N-1} \boldsymbol{h}(n) z^{-n}$ and let $p=r$ for simplicity. Assuming $\boldsymbol{h}(N-1) \neq \mathbf{0}$, the degree of $\boldsymbol{H}(z)$ is at least $N-1$; if the rank of $\boldsymbol{h}(N-1)$ is $\rho$, this degree is at least $(N-1) \rho$. Finally, if the degree of $\boldsymbol{H}(z)$ is equal to $N-1$, then $\boldsymbol{h}(N-1)$ has rank one.

\section{B. Properties of Lossless Transfer Matrices}

We now outline several features of lossless systems. Not all of these are proved here, for sake of brevity; however, appropriate references are included for the interested reader. We shall assume for this section and for the rest of the paper, that $c=1$ in (15) and (16).

Property 3.1-On the Autocorrelation Sequence of $\boldsymbol{h}(n)$ : The autocorrelation sequence corresponding to any impulse response is defined as

$$
\boldsymbol{r}(k)=\sum_{m=-\infty}^{\infty} \boldsymbol{h}^{\dagger}(m+k) \boldsymbol{h}(m)
$$

If $\boldsymbol{H}(z)$ is lossless, the autocorrelation of the impulse response satisfies $\boldsymbol{r}(k)=\delta(k) \boldsymbol{I}_{r}$. The proof follows by substituting $\boldsymbol{H}(z)=\sum_{n=-\infty}^{\infty} \boldsymbol{h}(n) z^{-n}$ into (16) and equating like powers of $z$ on both sides of the resulting equation.

Property 3.2-On the Determinant of a Square Lossless Transfer Matrix: Let $\boldsymbol{H}(z)$ be $p \times p$ and lossless. Then $\mathfrak{H}(z) \triangleq \operatorname{det} \boldsymbol{H}(z)$ is also lossless, i.e., stable all-pass! This follows from (16): take the determinant on both sides. Since $\operatorname{det} \boldsymbol{A}=\operatorname{det} \boldsymbol{A}^{T}$, this results in $\operatorname{det} \boldsymbol{H}_{*}(1 / z)$ det $\boldsymbol{H}(z)=1$, which simplifies to the all-pass property $\tilde{H}(z) \mathfrak{H}(z)=1$. Since $\mathfrak{H}(z)=P(z) / \mathcal{Q}(z)$, it is automatically stable. If $\boldsymbol{H}(z)$ is FIR, then $\mathcal{H C}(z)$ is a pure delay, i.e., $\mathfrak{H C}(z)=z^{-(K-1)}$.

Property 3.3-On the Normal Ranks of $\boldsymbol{P}(z)$ and $\boldsymbol{Q}(z)$ : Recall that, for any MFD, the normal rank (defined in Section III-A.5) of $\boldsymbol{Q}(z)$ is $\rho_{q}=p$ (otherwise the inverse in (31) will not exist for any $z$ !). If $\boldsymbol{H}(z)$ is lossless and if $p=r$, it turns out that the normal rank $\rho_{p}$ of $\boldsymbol{P}(z)$ is also equal to $p$. This can be seen as follows: since $p=r, \boldsymbol{H}^{T}(z)$ is also lossless. Accordingly, we have $\boldsymbol{H}(z)$ $\tilde{\boldsymbol{H}}(z)=\boldsymbol{I}_{p}$. Substituting $(31)$ and rearranging, we obtain

$$
\boldsymbol{P}(z) \tilde{\boldsymbol{P}}(z)=\boldsymbol{Q}(z) \tilde{\boldsymbol{Q}}(z) \text { for all } z .
$$

The matrix on the RHS of (43) is $p \times p$ and has rank $p$ for some $z$ ( since $\rho_{q}=p$.) For this same $z$, it is therefore clear that $\boldsymbol{P}(z)$ must have rank $p$, proving that $\rho_{p}=p$.

Property 3.4-Relation Between Poles and Zeros of Lossless $\boldsymbol{H}(z)$ when $p=r$ : It is well-known [24], [30] that, for scalar all-pass functions, poles and zeros occur in conjugate-reciprocal pairs, i.e., if $\alpha$ is a pole, then $1 / \alpha^{*}$ is a zero. For a $p \times p$ rational lossless system a similar result holds as proved next. Consider the irreducible representation (31). Since $\rho_{p}=\rho_{q}=p$, the poles of $\boldsymbol{H}(z)$ are given by the zeros of $Q(z)$ while the zeros of $\boldsymbol{H}(z)$ are given by the zeros of $\mathcal{P}(z)$ (see Section IIIA.5). If $\alpha$ is a pole, i.e., a zero of $Q(z)$, then there is a $p \times 1$ vector $\boldsymbol{v} \neq \mathbf{0}$ such that $\boldsymbol{v}^{T} \boldsymbol{Q}(\alpha)=\mathbf{0}$. This implies from (43), that either

$$
\boldsymbol{v}_{1}^{T} \triangleq \boldsymbol{v}^{T} \boldsymbol{P}(\alpha)=\mathbf{0}
$$

or

$$
\boldsymbol{v}_{1}^{T} \tilde{\boldsymbol{P}}(\alpha)=\mathbf{0}, \text { with } \boldsymbol{v}_{1} \neq \mathbf{0} .
$$

If (44) is true, it violates coprimeness of $\boldsymbol{Q}(z)$ and $\boldsymbol{P}(z)$ (see (41) and the comments preceding it). The only possibility then is (45) which implies $\operatorname{det} \boldsymbol{P}_{*}^{T}(1 / \alpha)=0$, i.e., det $\boldsymbol{P}\left(1 / \alpha^{*}\right)=0$. In summary, if $\alpha$ is a pole of $\boldsymbol{H}(z)$ then $1 / \alpha^{*}$ is a zero of $\boldsymbol{H}(z)$. This property is exactly analogous to that of a scalar all-pass function [24]. Conversely, suppose $\beta$ is a zero of $\boldsymbol{H}(z)$, i.e., a zero of $P(z)$. Then $\boldsymbol{v}_{2}^{T} \boldsymbol{P}(\beta)=\mathbf{0}$ for some $\boldsymbol{v}_{2} \neq \mathbf{0}$. In view of (43), we conclude by arguing in a manner similar to the above, that $1 / \beta^{*}$ is a zero of $\mathcal{Q}(z)$. So if $\beta$ is a zero, then $1 / \beta^{*}$ is a pole.

Since each zero $\beta$ of $\boldsymbol{H}(z)$ gives rise to a pole of $\boldsymbol{H}(z)$ at $1 / \beta^{*}$ we conclude that all the zeros of a lossless matrix $\boldsymbol{H}(z)$ are strictly outside the unit circle (since all the poles are strictly inside). Poles and zeros do not therefore overlap, for lossless functions! As a result, there cannot be any cancelling factors between $\rho(z)$ and $Q(z)$ as long as (31) is irreducible. In summary, the degree of $\boldsymbol{H}(z)$, which is the degree of $Q(z)$, is also equal to the degree of the allpass function $\mathfrak{H}(z)=\mathscr{P}(z) / \mathcal{Q}(z)$. This beautiful property is very special to lossless transfer matrices and is worth displaying as an equation:

$$
\operatorname{deg} \boldsymbol{H}(z)=\operatorname{deg} \mathfrak{H}(z), \quad \text { for lossless } \boldsymbol{H}(z) .
$$


In particular, if $\boldsymbol{H}(z)$ is also FIR, then from Property 3.2 (in Section III-B) we conclude that

$$
\operatorname{deg} \boldsymbol{H}(z)=\operatorname{deg} z^{-(K-1)}=K-1 .
$$

In other words, if $\boldsymbol{H}(z)$ is a $p \times p$ causal, FIR lossless system, its degree is simply the power $K-1$ of $z^{-1}$ appearing in $\mathfrak{H}(z)$.

Property 3.5-The Boundedness Property: A lossless transfer function $\boldsymbol{H}(z)$, which satisfies (15) on the unit circle by definition, satisfies the following property outside the unit circle:

$$
\boldsymbol{H}^{\dagger}(z) \boldsymbol{H}(z) \leq \boldsymbol{I}_{r}
$$

(See Section I for meaning of matrix inequality notations.) This property follows from the matrix version of maximum modulus theorem [27]. A direct proof of this theorem, based on energy balance arguments and linearsystem principles can be found in [26, Appendix A2].

The opposite inequality is satisfied inside the unit circle, i.e., $\boldsymbol{H}^{\dagger}(z) \boldsymbol{H}(z) \geq \boldsymbol{I}_{r}$, for $|z| \leq 1$. The proof for the cases $p=r$ follows by noting that (16) implies $\tilde{\boldsymbol{H}}(z)$ $=\boldsymbol{H}^{-1}(z)$, and using this relation in (47). For the case $p$ $>r$ the result remains true, even though the proof is trickier (construct a $p \times p$ lossless system $[\boldsymbol{H}(z) \boldsymbol{G}(z)]$ by augmenting the $p \times(p-r)$ system $\boldsymbol{G}(z)$, and apply the result for square lossless matrices). Details are omitted here for brevity. Summarizing, any lossless $\boldsymbol{H}(z)$ satisfies,

$$
\boldsymbol{H}^{\dagger}(z) \boldsymbol{H}(z)\left\{\begin{array}{cc}
=\boldsymbol{I}_{r} & |z|=1, \\
\leq \boldsymbol{I}_{r} & |z|>1, \\
\geq \boldsymbol{I}_{r} & |z|<1 .
\end{array}\right.
$$

For the scalar case, this relation is well-known [16, p. 494], [56] and finds application in frequency-transformations [24], [31]. See Property 3.10 for further comments. The inequalities $\geq$ and $\leq$ in (48) are replaced with strict inequalities $>$ and $<$ in the scalar case, unless $H(z)$ is a constant (this comment does not hold for the matrix case; see [26, Appendix A2]). One way to remember the result (48) is to note that the simple lossless system $z^{-1}$ satisfies (48) trivially.

Property 3.6-On Minimality of Orthogonal Implementations: Consider a discrete-time system implementation with state space description as in (36), (37). The implementation is said to be orthogonal if the matrix $\left[\begin{array}{cc}A & B \\ C & D\end{array}\right]$ is unitary. ${ }^{3}$ The significance of this property in the context of lossless systems is given in Property 3.7 below. Assuming that the system is stable (i.e., all eigenvalues of $\boldsymbol{A}$ have magnitude less than unity) and that $\boldsymbol{R}$ is unitary, we now show that the implementation is both controllable and observable. In other words (Section III-A.3), the implementation automatically has the smallest possible number of delays (or state variables)!

"Even though the term "unitary implementations" would be more appropriate, the term "orthogonal" has become more or less standard
In order to prove this result, we first show that the implementation is observable. Assume the contrary. This means (Section III-A.3) that there exists a vector $\boldsymbol{v} \neq \mathbf{0}$ such that

$$
\boldsymbol{A v}=\lambda \boldsymbol{v}
$$

and

$$
\boldsymbol{C v}=\mathbf{0} \text {. }
$$

The orthogonality of $\boldsymbol{R}$ implies in particular,

$$
\boldsymbol{A}^{\dagger} \boldsymbol{A}+\boldsymbol{C}^{\dagger} \boldsymbol{C}=I_{d} \text {. }
$$

Postmultiplying (51) with $v$ and using (50) results in

$$
\boldsymbol{A}^{\dagger} \boldsymbol{A} \boldsymbol{v}=\boldsymbol{v}, \quad \boldsymbol{v} \neq \mathbf{0}
$$

i.e., $\boldsymbol{v}^{\dagger} \boldsymbol{A}^{\dagger} \boldsymbol{A} \boldsymbol{v}=\boldsymbol{v}^{\dagger} \boldsymbol{v}$. Since (49) is also assumed to be true, we then have $|\lambda|^{2}=1$, which implies that there exists an eigenvalue of $\boldsymbol{A}$ whose magnitude is equal to unity. This violates the assumed stability of the implementation. The conclusion is that the implementation is observable. The proof of controllability is analogous, and will be omitted.

Property 3.7-On the Orthogonality of State-Space Realizations: In Section II we found simple examples of lossless systems based on constant unitary matrices. The connection between losslessness and constant unitary matrices is more fundamental, and is given by the following result: A stable transfer matrix $\boldsymbol{H}(z)$ is lossless if and only if there exists an implementation with minimum number of delays (i.e., $d=K-1=$ degree of $\boldsymbol{H}(z)$ ) whose state-space description (36), (37) is such that the system matrix defined by

$$
\boldsymbol{R} \triangleq\left[\begin{array}{ll}
\boldsymbol{A} & \boldsymbol{B} \\
\boldsymbol{C} & \boldsymbol{D}
\end{array}\right]
$$

is unitary. The history of this result dates back to certain theorems in classical, continuous-time scattering theory [5], [28], and to a result in system theory called the Kalman-Yakubovich lemma [5]. A direct proof is available in [29]; a simplified proof for the scalar case can be found in [54]. Variations of this result in the context of continuous-time passive network theory are given in [5].

Combining this result with Property 3.6 we conclude that whenever an implementation is stable and orthogonal, it represents a realization of a lossless system, which moreover has the smallest number of delays.

Property 3.8-On the Matrix of Impulse-Response Coefficients: Let $\boldsymbol{H}(z)$ be FIR so that $\boldsymbol{H}(z)=\Sigma_{n=0}^{N-1} \boldsymbol{h}(n) z^{-n}$. To fix ideas, let $N-1=2$ for example. Consider the matrix

$$
\boldsymbol{S}=\left[\begin{array}{lll}
\boldsymbol{h}(0) & \mathbf{0} & \mathbf{0} \\
\boldsymbol{h}(1) & \boldsymbol{h}(0) & 0 \\
\boldsymbol{h}(2) & \boldsymbol{h}(1) & \boldsymbol{h}(0) \\
\mathbf{0} & \boldsymbol{h}(2) & \boldsymbol{h}(1) \\
\mathbf{0} & \mathbf{0} & \boldsymbol{h}(2)
\end{array}\right]
$$


Each entry in this matrix is itself a $p \times r$ matrix. So for arbitrary $N, S$ is a $p(2 N-1) \times r N$ matrix. It turns out that this matrix is unitary (i.e., $S \boldsymbol{S}=\boldsymbol{I}_{r N}$ ) if and only if $\boldsymbol{H}(z)$ is lossless. The proof follows by using Property 3.1. Thus, the losslessness of $\boldsymbol{H}(z)$ manifests itself as the unitariness of two constant matrices; one is (53) corresponding to the family of orthogonal state-space descriptions and the other is (54).

Property 3.9-On the Mirror-Image Relation Between Numerator and Denominator: Scalar lossless functions, i.e., all-pass functions, can always be written [30], [54] in the form

$$
H(z)=z^{-K} e^{j \theta} \frac{b_{N}^{*}+b_{N-1}^{*} z^{-1}+\cdots+b_{0}^{*} z^{-N}}{b_{0}+b_{1} z^{-1}+\cdots+b_{N} z^{-N}} .
$$

In other words, except for the factor $z^{-K} e^{j \theta}$, the numerator is obtained by writing the denominator coefficients backwards and conjugating. In the case of lossless transfer matrices, a similar result does not exist. In fact, $Q(z)$ and $\boldsymbol{P}(z)$ do not necessarily have the same dimension, unless $p=r$. Moreover, for FIR lossless matrices, there is clearly no mirror image relation between $P(z)$ and $Q(z)$. From the examples of (21) and (29), these statements are even more clear.

Property 3.10-Frequency-Transformation Property: Given a transfer matrix $\boldsymbol{H}(z)$, if we replace each $z$ in its expression with $1 / A(z)$ where $A(z)$ is a stable allpass function, the operation is called a frequency transformation. The name originates from the fact [31], [24] that, such transformations can be used to convert low-pass filters into various other forms (such as, for example, bandpass functions). Recall that a stable all-pass function $A(z)$ satisfies a property analogous to $(48)$, i.e.,

$$
|A(z)| \begin{cases}=1 & |z|=1 \\ <1 & |z|>1 \\ >1 & |z|<1 .\end{cases}
$$

This ensures that the unit circle is mapped onto the unit circle during the transformation. Moreover the points inside and outside the unit circle are mapped, respectively, into points inside and outside. Consequently, the frequency-transformed version $\boldsymbol{G}(z)$ is stable if and only if $\boldsymbol{H}(z)$ is stable. If $\boldsymbol{H}(z)$ is lossless, then the transformed function $\boldsymbol{H}(1 / A(z))$ also remains lossless.

Property 3.11-Rank of the Impulse Response Coefficients: Let $\boldsymbol{H}(z)$ be FIR so that $\boldsymbol{H}(z)=\sum_{n=0}^{N-1} \boldsymbol{h}(n) z^{-n}$. Assume $\boldsymbol{h}(0) \neq \mathbf{0}$ and $\boldsymbol{h}(N-1) \neq \mathbf{0}$. With $p>r$ it is obvious that the rank of $\boldsymbol{h}(n)$ is less than $p$ for any $n$. If $\boldsymbol{H}(z)$ is lossless, the ranks of $\boldsymbol{h}(0)$ and $\boldsymbol{h}(N-1)$ are less than $p$ even if $p=r$. To see this note that by Property 3.1 , we have $\boldsymbol{r}(k)=\delta(k) \boldsymbol{I}_{r}$. In particular, this implies $\boldsymbol{h}^{\dagger}(N-1) \boldsymbol{h}_{0}=\mathbf{0}$, so that the ranks are less than $p$. So, for lossless FIR $\boldsymbol{H}(z)$ with $p=r, \boldsymbol{h}(0)$ and $\boldsymbol{h}(N-1)$ are singular matrices. For IIR lossless systems, however, $\boldsymbol{h}(0)$ can be nonsingular [example: $\boldsymbol{H}(z)=\left(a^{*}+\right.$ $\left.\left.z^{-1}\right) /\left(1+a z^{-1}\right) I_{p}\right]$.

\author{
IV. The Most General Form of Lossless \\ Transfer Matrices
}

Assuming that $\boldsymbol{H}(z)$ is lossless (with each entry $H_{k m}(z)$ a rational function ), is it possible to express it in a simple algebraic form? In this section we shall answer this question. From this it is possible to derive structures (i.e., interconnection of delays, multipliers, and adders) for implementation of lossless transfer matrices. Only causal systems will be considered ( $s$ o that $\boldsymbol{h}(n)=\mathbf{0}$ for $n<0$ ). The structures will have the following important features:

1) Any lossless transfer matrix can be realized by appropriate choice of multiplier values.

2) The number of scalar delay elements (or state variables) is the smallest required, viz., the McMillan degree.

3) The number of parameters used in the structure is the smallest necessary to characterize a lossless transfer matrix of a given degree.

One of the most influential references on continuoustime lossless systems is the classic text by Belevitch [4]. Many of the following discussions and results can be inferred by careful translation into the discrete-time domain of the excellent treatment in [4]. The following independent treatment, however, is entirely $z$-domain based. Please review the notations section (Section I) at this point for ease of reading.

\section{A. FIR Lossless Matrices of Arbitrary Degree with $p=$ $r=2$}

We shall first derive an expression for the most general form of a $2 \times 2$ causal FIR lossless system of arbitrary degree. We then show how such a matrix can be synthesized as a cascade of $2 \times 2$ elementary lossless building blocks. We shall first prove the following:

Lemma 4.1: The most general causal FIR $2 \times 2$ lossless system with arbitrary degree can be written in the form

$$
\boldsymbol{E}(z)=\left[\begin{array}{cc}
E_{00}(z) & c z^{-K} \tilde{E}_{10}(z) \\
E_{10}(z) & -c z^{-K} \tilde{E}_{00}(z)
\end{array}\right]
$$

where $E_{00}(z)$ and $E_{10}(z)$ satisfy the power complementary property, $c$ is a scalar constant with $|c|=1$, and $K$ is a large enough positive integer (to make the entries of the right column in (56) causal.

Note that (21) is an example of this form with $c=-1$, $K=1$. To prove the lemma, note that any lossless matrix

$$
\boldsymbol{E}(z)=\left[\begin{array}{ll}
E_{00}(z) & E_{01}(z) \\
E_{10}(z) & E_{11}(z)
\end{array}\right]
$$

satisfies $\tilde{\boldsymbol{E}}(z) \boldsymbol{E}(z)=\boldsymbol{I}$. This can equivalently be written as

$$
\begin{aligned}
& \tilde{E}_{00}(z) E_{00}(z)+\tilde{E}_{10}(z) E_{10}(z)=1, \\
& \tilde{E}_{00}(z) E_{01}(z)+\tilde{E}_{10}(z) E_{11}(z)=0, \\
& \tilde{E}_{01}(z) E_{01}(z)+\tilde{E}_{11}(z) E_{11}(z)=1 .
\end{aligned}
$$


Equation (57) implies that $E_{00}(z)$ and $E_{10}(z)$ do not share a common polynomial factor (because the RHS is a nonzero constant). Similarly $E_{11}(z)$ and $E_{01}(z)$ do not share a common factor [by (59)]. From these, coupled with (58), we conclude that $E_{11}(z)$ has all the polynomial factors contained in $\tilde{E}_{00}(z)$ and vice versa. Same is true of the pair $E_{01}(z)$ and $\tilde{E}_{10}(z)$. As a result, the four components of $\boldsymbol{E}(z)$ are related by

$$
\begin{aligned}
& E_{11}(z)=c_{1} z^{-K_{1}} \tilde{E}_{00}(z), \\
& E_{01}(z)=c_{2} z^{-K_{2}} \tilde{E}_{10}(z) .
\end{aligned}
$$

By using (60), (61) in (58) we conclude $c_{1}=-c_{2}$ and $K_{1}$ $=K_{2}$. By using (60) and (61) in (59) and comparing with (57) we obtain $\left|c_{1}\right|=1$ as well. Substituting these results in $\boldsymbol{E}(z)$, we see that it reduces to the form (56), completing the proof of Lemma 4.1.

In order to obtain a cascade-form realization for $E(z)$, note that $\boldsymbol{E}(z)$ in (56) is essentially determined by the 0 th column. The 0 th column represents a power-complementary pair $\left[E_{00}(z), E_{10}(z)\right]$. The best strategy is to derive a cascade-form realization of the 0 th column ${ }^{4}$ which can then be used to implement the entire $2 \times 2$ matrix $\boldsymbol{E}(z)$. We shall obtain the realization for the case of a real-coefficient matrix, just for simplicity. For this purpose we use a result from [13, p. 1047] stated below.

Lemma 4.2: Let $P_{m}(z)$ and $Q_{m}(z)$ be real-coefficient FIR systems with

$$
P_{m}(z)=\sum_{n=0}^{m} p_{m}(n) z^{-n}, \quad Q_{m}(z)=\sum_{n=0}^{m} q_{m}(n) z^{-n}
$$

with $p_{m}(0) p_{m}(m) \neq 0$. Assume that $\left|P_{m}\left(e^{j \omega}\right)\right|^{2}+$ $\left|Q_{m}\left(e^{j \omega}\right)\right|^{2}=1$. Then there exists another pair of realcoefficient transfer functions

$$
\begin{aligned}
& P_{m-1}(z)=\sum_{n=0}^{m-1} p_{m-1}(n) z^{-n}, \\
& Q_{m-1}(z)=\sum_{n=0}^{m-1} q_{m-1}(n) z^{-n}
\end{aligned}
$$

and a real $\theta_{m}$ such that

$$
\left[\begin{array}{l}
P_{m}(z) \\
Q_{m}(z)
\end{array}\right]=\left[\begin{array}{cc}
\cos \theta_{m} & \sin \theta_{m} \\
-\sin \theta_{m} & \cos \theta_{m}
\end{array}\right]\left[\begin{array}{l}
P_{m-1}(z) \\
z^{-1} Q_{m-1}(z)
\end{array}\right] .
$$

The consequence of this lemma is that we can implement the power complementary pair $\left[P_{m}(z), Q_{m}(z)\right]$ in terms of the lower-degree pair $\left[P_{m-1}(z), Q_{m-1}(z)\right]$ and the $2 \times 2$ orthogonal matrix in (64a). The lower degree pair is power-complementary [as can be proved by inverting (64a)]. Repeated application of this idea results in the cascade-form implementation of Fig. 4(a) for $\left[P_{m}(z)\right.$,

${ }^{4}$ Note that the degree of a $p \times 1$ system is equal to the degree of the highest-degree element in the column vector.

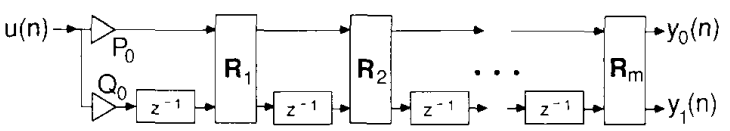

(a)

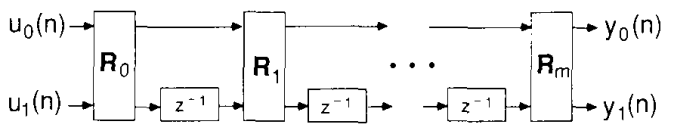

(b)

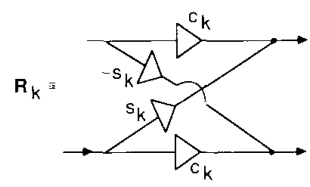

(c)

Fig. 4. (a) Cascaded lattice structure for lossless

$$
\left[\begin{array}{l}
P_{m}(z) \\
Q_{m}(z)
\end{array}\right]
$$

where $P_{m}(z)=Y_{0}(z) / U(z), Q_{m}(z)=Y_{1}(z) / U(z)$. (b) Cascaded lattice structure for lossless $\boldsymbol{H}_{m j}(z)$ where

$$
\left[\begin{array}{l}
Y_{0}(z) \\
Y_{1}(z)
\end{array}\right]=\boldsymbol{H}_{m}(z)\left[\begin{array}{l}
U_{0}(z) \\
U_{1}(z)
\end{array}\right]
$$

(c). Details of the matrix $\boldsymbol{R}_{k}$. Here $c_{k}=\cos \theta_{k}, s_{k}=\sin \theta_{k}$

$Q_{m}(z)$ ], called the cascaded FIR lattice structure. We thus have

$$
\left[\begin{array}{c}
P_{m}(z) \\
Q_{m}(z)
\end{array}\right]=\boldsymbol{R}_{m} \mathbf{\Lambda}(z) \boldsymbol{R}_{m-1} \mathbf{\Lambda}(z) \cdots \boldsymbol{R}_{\mathbf{1}} \mathbf{\Lambda}(z)\left[\begin{array}{c}
P_{0} \\
Q_{0}
\end{array}\right]
$$

where $P_{0}, Q_{0}$ are constants with

$$
P_{0}^{2}+Q_{0}^{2}=1, \quad \boldsymbol{R}_{k}=\left[\begin{array}{cc}
\cos \theta_{k} & \sin \theta_{k} \\
-\sin \theta_{k} & \cos \theta_{k}
\end{array}\right]
$$

and

$$
\mathbf{\Lambda}(z)=\left[\begin{array}{ll}
1 & 0 \\
0 & z^{-1}
\end{array}\right]
$$

Notice the fundamental difference between this structure and the standard linear-prediction lattice [50], [51] which does not generate power-complementary transfer functions (also see Section V of [13]).

Now assume that we have a $2 \times 2$ causal FIR realcoefficient lossless system of the form

$$
\boldsymbol{H}_{m}(z)=\left[\begin{array}{cc}
P_{m}(z) & c z^{-K} \tilde{Q}_{m}(z) \\
Q_{m}(z) & -c z^{-K} \tilde{P}_{m}(z)
\end{array}\right]
$$

where $P_{m}(z)$ and $Q_{m}(z)$ are as in (62). We know the 0th column can be implemented as in (64a). By substituting (64a) in (65) and simplifying, it can be shown that the entire matrix (65) can be rewritten as

$$
\boldsymbol{H}_{m}(z)=\left[\begin{array}{cc}
\cos \theta_{m} & \sin \theta_{m} \\
-\sin \theta_{m} & \cos \theta_{m}
\end{array}\right] \mathbf{\Lambda}(z) \boldsymbol{H}_{m-1}(z)
$$


where

$$
\begin{aligned}
\boldsymbol{H}_{m-1}(z)=\left[\begin{array}{cc}
P_{m-1}(z) & c z^{-(K-1)} \tilde{Q}_{m-1}(z) \\
Q_{m-1}(z) & -c z^{-(K-1)} \tilde{P}_{m-1}(z)
\end{array}\right] \\
K>m
\end{aligned}
$$

Clearly $\boldsymbol{H}_{m-1}(z)$ is a real-coefficient lossless matrix. This is called the remainder system, after extracting the $2 \times 2$ lossless building block from the given system $\boldsymbol{H}_{m}(z)$. In (65) $K \geq m$ for causality. If $K>m$ we can factorize the quantity $z^{-K+m}$ and convert the problem into the $K=m$ case. Assuming therefore that $K=m$, we see that after $m$ repetitions of the above extraction process we obtain a realization for $\boldsymbol{H}_{m}(z)$ of the form

$$
\boldsymbol{H}_{m}(z)=\boldsymbol{R}_{m} \boldsymbol{\Lambda}(z) \boldsymbol{R}_{m-1} \boldsymbol{\Lambda}(z) \cdots \boldsymbol{R}_{1} \boldsymbol{\Lambda}(z) \boldsymbol{R}_{0}
$$

where $\boldsymbol{R}_{k}$ are $2 \times 2$ orthogonal matrices and $\boldsymbol{\Lambda}(z)$ is as in $(64 \mathrm{c})$. This gives us the cascaded lattice realization of Fig. 4(b) for the $2 \times 2$ real-coefficient FIR lossless matrix. Several examples of this form can be found in [13], [19].

\section{B. FIR Lossless Transfer Matrices of Degree One}

Next we consider degree-one systems with $p=r$ where $p$ is arbitrary. Thus, $\boldsymbol{H}(z)$ is a square matrix representing a FIR system. As the degree is one, there is only one scalar delay in the structure, so that $\boldsymbol{H}(z)$ takes the form

$$
\boldsymbol{H}(z)=\boldsymbol{h}(0)+z^{-1} \boldsymbol{h}(1) .
$$

To avoid trivialities, we assume $\boldsymbol{h}(1) \neq \mathbf{0}$ so that the degree of $\boldsymbol{H}(z)$ is strictly equal to one. With degree equal to one, the matrices $\boldsymbol{h}(0)$ and $\boldsymbol{h}(1)$ satisfy additional conditions. For example, we cannot have $\boldsymbol{h}$ (1) $=\boldsymbol{I}_{r}$ (unless $r=1$ ) because this would require the use of $r$ scalar delays for implementation.

Let us be more precise on this. As $\boldsymbol{H}(z)$ is FIR and causal, the poles are at $z=0$. Assume that we have written down the state-space equations (36), (37) for the implementation. Since the degree is one, $\boldsymbol{A}$ is a scalar $(1 \times$ 1 matrix ). The eigenvalues of $\boldsymbol{A}$ correspond to the poles of $\boldsymbol{H}(z)$. So the only possible $\boldsymbol{A}$ matrix is $\boldsymbol{A}=0$. From (38) we therefore obtain

$$
\boldsymbol{h}(0)=\boldsymbol{D}, \quad \boldsymbol{h}(1)=\boldsymbol{C B} .
$$

The matrix $B$ is $1 \times p$ (row vector) and $C$ is $p \times 1$ so that $\boldsymbol{C B}$ has rank equal to one. So the rank of $\boldsymbol{h}(1)$ is one. Next, from Property 3.11 we know that the rank of $\boldsymbol{h}(0)$ is necessarily less than $p$.

Having made these preliminary conclusions, recall now that $\boldsymbol{H}(z)$ is unitary on the unit circle [i.e., satisfies (15)]. In particular, setting $z=1$ ( or $\omega=0$ ), we see that $\boldsymbol{H}$ (1) is required to be unitary. We can therefore conveniently express $\boldsymbol{H}(z)$ in the form

$$
\boldsymbol{H}(z)=\left(1-z^{-1}\right) \boldsymbol{S}+\boldsymbol{R}
$$

where $\boldsymbol{S}$ is $p \times p$, and $\boldsymbol{R}$ is $p \times p$ unitary. If we use this form and now impose the condition $\boldsymbol{H}(z) \tilde{H}(z)=\boldsymbol{I}$ (which is a requirement for any square lossless matrix), we find after simplification

$$
\begin{aligned}
\mathbf{0}= & 2 \boldsymbol{S} \boldsymbol{S}^{\dagger}+\boldsymbol{S} \boldsymbol{R}^{\dagger}+\boldsymbol{R} \boldsymbol{S}^{\dagger}-z^{-1}\left(\boldsymbol{S} \boldsymbol{S}^{\dagger}+\boldsymbol{S} \boldsymbol{R}^{\dagger}\right) \\
& -z\left(\boldsymbol{S} \boldsymbol{S}^{\dagger}+\boldsymbol{R} \boldsymbol{S}^{\dagger}\right) .
\end{aligned}
$$

Equating like powers of $z$ we obtain the following two necessary conditions for losslessness:

$$
\begin{aligned}
\boldsymbol{S} \boldsymbol{S}^{\dagger}+\boldsymbol{S} \boldsymbol{R}^{\dagger} & =\mathbf{0}, \\
2 \boldsymbol{S} \boldsymbol{S}^{\dagger}+\boldsymbol{S} \boldsymbol{R}^{\dagger}+\boldsymbol{R} \boldsymbol{S}^{\dagger} & =\mathbf{0} .
\end{aligned}
$$

Since $\boldsymbol{S} \boldsymbol{S}^{\dagger}$ is Hermitian, (74) automatically restricts $\boldsymbol{S} \boldsymbol{R}^{\dagger}$ to be Hermitian. As a result, (75) is equivalent to $S S^{\dagger}+$ $\boldsymbol{S} \boldsymbol{R}^{\dagger}=\mathbf{0}$, which in turn is (74) itself. In summary the condition (16) can be met simply by forcing the single relation (74). Using (74), we rewrite (72) as

$$
\boldsymbol{H}(z)=\left[\boldsymbol{I}-\boldsymbol{S} \boldsymbol{S}^{\dagger}+z^{-1} \boldsymbol{S} \boldsymbol{S}^{\dagger}\right] \boldsymbol{R} .
$$

Comparing (70) and (76) we see that $\boldsymbol{h}(1)=S S^{\dagger} \boldsymbol{R}$. Here $\boldsymbol{R}$ has full rank and $\boldsymbol{h}(1)$ has rank one, so that $\boldsymbol{S} \boldsymbol{S}^{\dagger}$ must have rank one! Since $\boldsymbol{S S}^{\dagger}$ is Hermitian with rank one, it can always be rewritten [32] in the form

$$
S S^{\dagger}=v v^{\dagger}
$$

for appropriate choice of a column vector $\boldsymbol{v}$. (Matrices of the form (77) are called diadics.) Thus, (76) reduces to the form

$$
\boldsymbol{H}(z)=\left[\boldsymbol{I}-\boldsymbol{v} \boldsymbol{v}^{\dagger}+z^{-1} \boldsymbol{v} \boldsymbol{v}^{\dagger}\right] \boldsymbol{R} .
$$

It can be shown further that the norm of $v$ is unity i.e., $\boldsymbol{v}^{\dagger} \boldsymbol{v}=1$. For this note that the quantity inside square brackets in (78) is lossless, and in particular is unitary for $z=-1$. In other words $I-2 v v^{\dagger}$ is unitary; since this is at the same time Hermitian [32], its eigenvalues are restricted to be \pm 1 . One of the eigenvalues of this matrix is $\left(1-2 \boldsymbol{v}^{\dagger} \boldsymbol{v}\right)$; this quantity is -1 iff $\boldsymbol{v}^{\dagger} \boldsymbol{v}=1$, and is 1 iff $\boldsymbol{v}^{\dagger} \boldsymbol{v}=0$. Since $\boldsymbol{v}=\mathbf{0}$ implies zero-degree systems [see (78)], the only possibility is $\boldsymbol{v}^{\dagger} \boldsymbol{v}=1$. Summarizing, we have as follows:

Lemma 4.3: If $\boldsymbol{H}(z)$ is a causal $p \times p$ FIR lossless matrix of degree one, then it must have the form (78) where $v$ is $p \times 1$ (i.e., a column vector) with unit norm, and $\boldsymbol{R}$ is a $p \times p$ unitary matrix. Conversely, any FIR matrix of the form (78) where $\boldsymbol{R}$ and $\boldsymbol{v}$ satisfy these conditions is necessarily lossless of degree one.

\section{FIR Lossless Matrices with Arbitrary Degree}

We now come to $p \times p$ FIR lossless matrices with degree $K-1>1$. It is clear that if we interconnect several systems of the form (78) in a cascade, then the resulting transfer matrix is the product of individual ones, and is hence lossless. In this way, examples of lossless FIR systems of arbitrary degree can be obtained. A less obvious result of considerable importance is the following: every causal $p \times p$ lossless FIR transfer matrix can be realized as such a cascade! This result, which we shall now prove, gives us a complete tool for synthesizing and implementing arbitrary rational FIR lossless systems. 
Suppose $\boldsymbol{H}_{m}(z)$ is a $p \times p$ causal FIR lossless transfer matrix of degree $m$. We will show that it can be written in the form

$$
\boldsymbol{H}_{m}(z)=\left[\boldsymbol{I}-\boldsymbol{v}_{m} \boldsymbol{v}_{m}^{\dagger}+\boldsymbol{v}_{m} \boldsymbol{v}_{m}^{\dagger} z^{-1}\right] \boldsymbol{H}_{m-1}(z)
$$

where $\boldsymbol{H}_{m-1}(z)$ is a $p \times p$ causal FIR lossless transfer matrix of degree $m-1$ and where $\boldsymbol{v}_{m}$ is a $p \times 1$ column vector of unit norm. In other words, there exist $\boldsymbol{v}_{m}$ and $\boldsymbol{H}_{m-1}(z)$ with these properties, such that $\boldsymbol{H}_{m}(z)$ can be implemented as in Fig. 5. We can now repeat the process by expressing $\boldsymbol{H}_{m-1}(z)$ in terms of an appropriate vector $\boldsymbol{v}_{m-1}$ and a lossless matrix $\boldsymbol{H}_{m-2}(z)$. Repeated application of this process terminates with the lossless transfer matrix $\boldsymbol{H}_{0}(z)$ of degree one, i.e., a constant unitary matrix. Fig. $6(a)$ is the overall appearance of the implementation which would result from this procedure, where

$$
\boldsymbol{G}_{m}(z) \triangleq \boldsymbol{I}-\boldsymbol{v}_{m} \boldsymbol{v}_{m}^{\dagger}+\boldsymbol{v}_{m} \boldsymbol{v}_{m}^{\dagger} z^{-1}
$$

An implementation of the degree-one building block $\boldsymbol{G}_{m}(z)$ is shown in Fig. 6(b). The quantity $\boldsymbol{H}_{m-1}(z)$ is said to be the remainder of a degree-reduction process, which involves extraction of the building block $\boldsymbol{G}_{m}(z)$ as described above.

It only remains to show how to construct $\boldsymbol{H}_{m-1}(z)$ from $\boldsymbol{H}_{m}(z)$ satisfying the above properties. Since $\boldsymbol{v}_{m}^{\dagger} \boldsymbol{v}_{m}=1$, (80) is clearly lossless, so $\boldsymbol{G}_{m}^{-1}(z)=\tilde{\boldsymbol{G}}_{m}(z)$ [see (16)]. As a result, (79) is equivalent to

$$
\left[\boldsymbol{I}-\boldsymbol{v}_{m} \boldsymbol{v}_{m}^{\dagger}+\boldsymbol{v}_{m} \boldsymbol{v}_{m}^{\dagger} z\right] \boldsymbol{H}_{m}(z)=\boldsymbol{H}_{m-1}(z)
$$

From (81) we see that if we start with FIR $\boldsymbol{H}_{m}(z)$, then $\boldsymbol{H}_{m-1}(z)$ is FIR. For causal $\boldsymbol{H}_{m}(z)$, the system $\boldsymbol{H}_{m-1}(z)$ is causal if and only if

$$
\boldsymbol{v}_{m} \boldsymbol{v}_{m}^{\dagger} \boldsymbol{h}_{m}(0)=\mathbf{0}
$$

where $\boldsymbol{h}_{m}(0)$ is the 0 th impulse response coefficient of $\boldsymbol{H}_{m}(z)$. Since $\boldsymbol{h}_{m}(0)$ is singular (Property 3.11 ) we know there exists a vector $v_{m}$ with unit norm such that (82) is true. Assume that $\boldsymbol{v}_{m}$ has been so chosen. Since $\boldsymbol{G}_{m}(z)$ (and hence $\tilde{\boldsymbol{G}}_{m}(z)$ ) and $\boldsymbol{H}_{m}(z)$ are unitary on the unit circle, so is $\boldsymbol{H}_{m-1}(z)$ as seen from (81). In summary, with $\boldsymbol{v}_{m}$ chosen to satisfy (82), the matrix $\boldsymbol{H}_{m-1}(z)$ is causal and FIR (hence stable) and is unitary on the unit circle; in other words, $\boldsymbol{H}_{m-1}(z)$ is guaranteed to be causal, FIR, and lossless. It only remains to check the degree of $\boldsymbol{H}_{m-1}(z)$. We can draw conclusions about the degree by taking determinants on both sides of (81):

$$
\begin{aligned}
\operatorname{det} & {\left[\boldsymbol{I}-\boldsymbol{v}_{m} \boldsymbol{v}_{m}^{\dagger}+\boldsymbol{v}_{m} \boldsymbol{v}_{m}^{\dagger} z\right] \operatorname{det} \boldsymbol{H}_{m}(z) } \\
& =\operatorname{det} \boldsymbol{H}_{m-1}(z) .
\end{aligned}
$$

By using Property 3.2 and (46b) we conclude

$$
z^{1} z^{-m}=z^{-n}
$$

where $n$ is the degree of $\boldsymbol{H}_{m-1}(z)$. Clearly $n=m-1$.

In conclusion, if we are given a causal $p \times p$ FIR lossless matrix $\boldsymbol{H}_{m}(z)$ of degree $m$, and if we define $\boldsymbol{H}_{m-1}(z)$ as in (81) where $\boldsymbol{v}_{m}$ is any column vector satisfying (82), then $\boldsymbol{H}_{m-1}(z)$ is a causal $p \times p$ FIR lossless matrix of

$$
H_{m-1}(z) \Rightarrow G_{m}(z) \rightarrow H_{m}(z)
$$

Fig. 5. Extraction of a degree-one FIR lossless section $\boldsymbol{G}_{m}(z)$ from lossless $\boldsymbol{H}_{m}(z)$ of degree $m$.
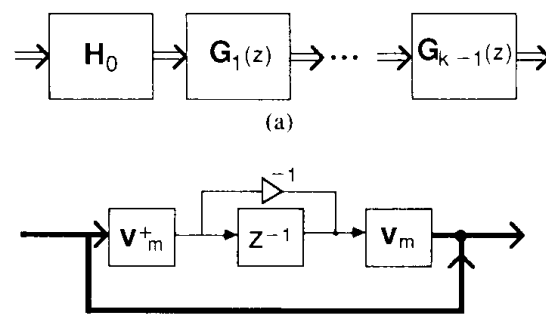

(b)

Fig. 6. (a) The overall appearance of the cascaded FIR structure, which has the transfer matrix $\boldsymbol{H}_{K-1}(z)$. Here each $\boldsymbol{G}_{m}(z)$ is a degree-one FIR lossless system, and $\boldsymbol{H}_{0}$ is a constant unitary matrix. (b) Implementation of $G_{m}(z)$.

degree $m-1$. Repeated application of this result leads to the following:

Theorem 4.1: Any causal $p \times p$ FIR lossless matrix $H_{K-1}(z)$ of degree $K-1$ can be expressed as a product of the degree-one lossless sections, i.e.,

$$
\begin{aligned}
\boldsymbol{H}_{K-1}(z)= & {\left[\boldsymbol{I}-\boldsymbol{v}_{K-1} \boldsymbol{v}_{K-1}^{\dagger}+\boldsymbol{v}_{K-1} \boldsymbol{v}_{K-1}^{\dagger} z^{-1}\right] \cdots } \\
& {\left[\boldsymbol{I}-\boldsymbol{v}_{2} \boldsymbol{v}_{2}^{\dagger}+\boldsymbol{v}_{2} \boldsymbol{v}_{2}^{\dagger} z^{-1}\right] } \\
& \cdot\left[\boldsymbol{I}-\boldsymbol{v}_{1} \boldsymbol{v}_{1}^{\dagger}+\boldsymbol{v}_{1} \boldsymbol{v}_{1}^{\dagger} z^{-1}\right] \boldsymbol{H}_{0}
\end{aligned}
$$

where $\boldsymbol{H}_{0}$ is a $p \times p$ constant unitary matrix, and $\boldsymbol{v}_{m}$ has unit norm.

Notice that many of the properties stated in Section III-B have been used in the above synthesis procedure!

Case of Real Coefficients: In theorem 4.1 if $\boldsymbol{H}_{K-1}(z)$ has real coefficients (i.e., it is LBR), then the vectors $\boldsymbol{v}_{m}$ turn out to be real. This can be seen by noting that if $h_{m}(0)$ is real and singular then we can find a real nonzero vector $\boldsymbol{v}_{m}$ satisfying (82). If $\boldsymbol{H}_{m}(z)$ is LBR and if $\boldsymbol{v}_{m}$ is real then $\boldsymbol{H}_{m-1}(z)$ obtained from (81) is LBR so that the reasoning can be repeated to prove that all the vectors $\boldsymbol{v}_{n}$ and the matrix $\boldsymbol{H}_{0}$ in the factorization (85) are real.

Unitariness of the System Matrix $\boldsymbol{R}$ : In Section III we mentioned that for lossless systems there exist structures such that the system matrix $\boldsymbol{R}$ defined in (53) is lossless. It turns out that if we implement $\boldsymbol{H}_{K-1}(z)$ as in Fig. 6(a) with each building block as in Fig. $6(\mathrm{~b})$ then the system matrix $\boldsymbol{R}$ is indeed unitary. A proof of this statement can be found in [59]. For this reason, the structure of Fig. 6 can be considered to be a unitary realization (more commonly called an orthogonal realization) of the lossless system $\boldsymbol{H}_{K-1}(z)$.

Eigenvalues of Lossless Systems: If $\boldsymbol{H}(z)$ is lossless then $\boldsymbol{H}\left(e^{j \omega}\right)$ is unitary so that all its eigenvalues have the same magnitude $\alpha>0$ for all $\omega$. In other words, these eigenvalues are allpass functions. As an example consider the degree-one lossless system (80). It can be verified that the set of $p$ eigenvalues of this system are: $\left(z^{-1}, 1,1\right.$, 
$\cdots, 1)$. To see this note that if we postmultiply (80) with $\boldsymbol{v}_{m}$ then the right hand side becomes $z^{-1} \boldsymbol{v}_{m}$ so that $\boldsymbol{v}_{m}$ is the eigenvector corresponding to the eigenvalue $z^{-1}$. On the other hand, if we postmultiply ( 80 ) with any vector $v$ orthogonal to $\boldsymbol{v}_{m}$ then the result is $v$ which shows that $\boldsymbol{v}$ is an eigenvector corresponding to the eigenvalue of unity.

IIR Lossless Matrices with Arbitrary Degree: In (85), if we replace the delay $z^{-1}$ in each degree-one lossless section by a stable all-pass function of the form

$$
A_{m}(z)=\frac{a_{m}^{*}+z^{-1}}{1+a_{m} z^{-1}}, \quad\left|a_{m}\right|<1
$$

these sections remain lossless (property 3.10). As a result, the cascade (85) is lossless, but is now IIR with poles at $z=-a_{m}$. This is essentially an obvious result, but more is true. It can be shown that any $p \times p$ IIR lossless transfer matrix of degree $K-1$ can be written in the form (85) provided each of the $K-1$ delays is replaced by an appropriate all-pass function of the form (86). The details of the synthesis procedure are omitted here for sake of brevity.

Finally, it should be mentioned that structures for rectangular FIR lossless matrices have been developed in [60], but the details are beyond the scope of our discussion here.

\section{Degrees of Freedom}

How many independent scalar numbers or parameters are needed to completely specify a lossless system? Losslessness, which is a constraint on the behavior of $\boldsymbol{H}(z)$ on the unit circle, reduces the number of degrees of freedom that would be normally available to specify a transfer function. For example, consider a scalar transfer function $H(z)=\left(a_{0}+a_{1} z^{-1}\right) /\left(1+b_{1} z^{-1}\right)$ of degree 1. As such there are three independent complex numbers (i.e., six freedoms). If we constrain $H(z)$ to be all-pass it has the form $e^{j \theta}\left(a^{*}+z^{-1}\right) /\left(1+a z^{-1}\right)$ which has only three freedoms ( $\theta$ is real and $a$ is complex).

In order to make more general statements, first consider the general $p \times p$ FIR lossless matrix of degree $K-1$. This is a cascade of the form in (85). Each vector $v_{m}$ has $p$ components (in general complex) which are constrained by the equation $\boldsymbol{v}_{m}^{\dagger} \boldsymbol{v}_{m}=1$. The equality constraint takes away one freedom, so this leaves a total of $2 p-1$ freedoms for $\boldsymbol{v}_{m}$, for each $m$.

Next we have to count the freedoms available in specifying $\boldsymbol{H}_{0}$. An arbitrary constant $p \times p$ matrix has $p^{2}$ complex freedoms (i.e., $2 p^{2}$ freedoms). Due to unitary constraints, the number of freedoms is reduced. It can be shown [33] that there are only $p^{2}$ freedoms in specifying a unitary matrix. As a result, the total number of parameters required to characterize a $p \times p$ lossless FIR matrix is equal to

$$
N_{f}=(K-1)(2 p-2)+p^{2} \text {. }
$$

Next consider the special case of real-coefficient lossless systems (i.e., LBR systems). Now the vector $\boldsymbol{v}_{m}$ is real and satisfies the constraint $\boldsymbol{v}_{m}^{T} \boldsymbol{v}_{m}=1$ so that it has only $p-1$ freedoms. The matrix $\boldsymbol{H}_{0}$ is real orthogonal so that it has only $\left(\begin{array}{l}p \\ 2\end{array}\right)$ freedoms [33]. The total number of freedoms is therefore

$$
N_{f, \text { real }}=(K-1)(p-1)+\left(\begin{array}{l}
p \\
2
\end{array}\right) .
$$

For the IIR case, if $K-1$ is the McMillan degree, we have $K-1$ poles coming from the all-pass sections (86) which are used to replace the delays in (85). Each of these poles can be complex and has two freedoms. So we should add $(2 K-2)$ to $(87)$, in order to obtain the total number of freedoms. For the real-coefficient case, the poles are either real or complex-conjugate pairs, so we add $K-1$ to $(88)$ to obtain the total number of freedoms.

\section{Applications}

In the last few sections, several properties and synthesis procedures for lossless systems were discussed. There have been several applications of these concepts in modern signal processing. As these applications are well-documented in the literature, this section will only act as a pointer to some of these.

\section{A. Low Sensitivity Digital Filter Design}

Consider a scalar transfer function $H(z)=P(z) / Q(z)$ with $P(z), Q(z)$ as in (30). Many structures exists for its implementation [24], [25] such as the direct form, parallel form, and the cascade form. It is well-known [24] that for large $N$, the direct form has very poor sensitivity properties, i.e., a small perturbation of $p_{n}, q_{n}$ (caused by digitization) results in large deviation of the frequency response from the ideal. Sometimes, it is possible for the digitized filter to become unstable even though the infinite-precision filter is stable.

An additional problem in a practical implementation of a digital filter is the possibility of limit cycles, which are oscillations caused by state-variable quantization. These are very undesirable, as they result in nonzero steady-state output in response to zero input. Such zero-input limit cycles must be suppressed in any good implementation. State-variable quantization also generates roundoff noise which propagates to the filter output via a noise transfer function. The noise gain [24] (which is an appropriate norm of the noise transfer function) depends on the polelocation for most standard structures; as a rule of thumb, this gain is larger for filters with poles crowded together and close to the unit circle.

The concept of losslessness plays a very crucial role [9]-[14], [49] in the design of digital filter structures, which have low sensitivity and noise gain, and which in addition are free from limit cycles. One of the simplest such structures is shown in Fig. 7 where $A_{0}(z)$ and $A_{1}(z)$ are stable all-pass functions (lossless functions). $H_{0}(z)$ is the main transfer function of interest, while $H_{1}(z)$ is an 


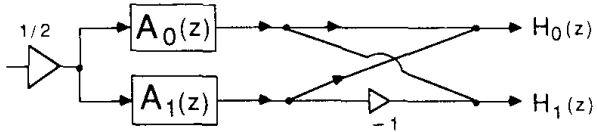

Fig. 7. Realization of transfer functions as a sum ano a difference of two all-pass functions.

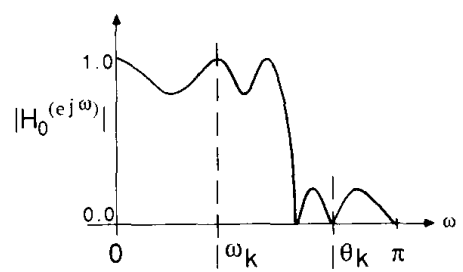

Fig. 8. Typical frequency-response magnitude for the transfer function $H_{0}(z)$.

auxiliary transfer function whose presence can be ignored until further notice.

In Fig. $7, H_{0}(z)$ is a sum of two all-pass functions. There is a surprising result [12], [34], [35] which says that many of the standard IIR tranfer functions (such as Butterworth, Chebyshev, and Elliptic [24]) can be expressed in this form. As a result, the structure of Fig. 7 is not as restricted as it looks at first sight. Qualitatively, the structure works as follows: for steady-state frequencies we have $A_{0}\left(e^{j \omega}\right)=e^{j \phi_{0}(\omega)}$ and $A_{1}\left(e^{j \omega}\right)=e^{j \phi_{1}(\omega)}$. Whenever the phases $\phi_{0}(\omega)$ and $\phi_{1}(\omega)$ are aligned, the magnitude of $H_{0}\left(e^{j \omega}\right)$ attains its maximum of unity. This happens at the extremal frequencies $\omega_{k}$ of the passband (see Fig. 8 for demonstration). When the phases $\phi_{0}(\omega)$ and $\phi_{1}(\omega)$ differ by (an odd integral multiple of) $\pi$, the magnitude of $H_{0}\left(e^{j \omega}\right)$ attains its lowest value (equal to zero). These correspond to the transmission-zeros $\theta_{k}$ of the stopband.

There exist several ways to implement the all-pass filters [16], [30], [36]-[39], in such a way that these remain stable allpass inspite of digitization of multipliers. These implementations are said to be structurally lossless. Notable among these is the Gray-Markel lattice structure [37][39]. With such an implementation, $H_{0}(z)$ is guaranteed to be stable and bounded in magnitude by unity for all $\omega$. Such implementations are said to be structurally bounded, and this property has been found to be the key requirement [11] for low-sensitivity structures. ${ }^{5}$ Examples and simulation results can be found in [11], [12], [40]. Once we implement $H_{0}(z)$, we can obtain $H_{1}(z)$ almost for free, just by subtracting (rather than adding) the outputs of the two all-pass filters.

A second advantage of the use of Gray-Markel lattice structures is that, inspite of coefficient quantization, the structure remains stable as long as the coefficients remain bounded by unity. Moreover, because of the losslessness of internal computational building blocks, the structure is free from limit cycles [39], [29], [14]. Finally, the lattice

${ }^{5}$ A stable transfer function $H_{0}(z)$ with $\left|H_{0}\left(e^{j \omega}\right)\right| \leq 1$ for all $\omega$ is called a bounded transfer function (bounded real or BR if, in addition, the coefficients are real). structure has the lowest possible roundoff noise gain (and is independent of pole locations) [54] among all all-pass structures of a given order. All these advantages arise because of the fact that, the lattice structure is a classic example of a system for which the matrix (53) is unitary. It can be shown [14], [29] that unitariness of (53) can be used as a tool to prove the above mentioned good features. Further advantages of the lattice structure include high modularity and pipelineability [41] which are attractive from a VLSI designer's viewpoint.

Complementary Properties: From Fig. 7 we have

$$
H_{0}(z)=\frac{A_{0}(z)+A_{1}(z)}{2}, \quad H_{1}(z)=\frac{A_{0}(z)-A_{1}(z)}{2}
$$

from which we deduce

$$
H_{0}(z)+H_{1}(z)=A_{0}(z)
$$

and

$$
\left|H_{0}\left(e^{j \omega}\right)\right|^{2}+\left|H_{1}\left(e^{j \omega}\right)\right|^{2}=1 .
$$

From (91) we see that $H_{0}(z), H_{1}(z)$ is a power complementary pair. Property $(90)$ which says that $H_{0}(z)$ and $H_{1}(z)$ add up to an all-pass function is called all-pass complementary property. Jointly, (90) and (91) are referred to as the doubly-complementary property.

In the case of FIR filters, it is once again possible to obtain low sensitivity structures by using the losslessness concept. A new class of lattice structures is reported in [13], for this purpose.

\section{B. Filter Bank Applications}

A digital filter bank is a collection of $M$ bandpass filters $H_{k}(z)$ which split a signal $x(n)$ into $M$ subbands. These subband signals are typically decimated (i.e., undersampled) by a factor of $M$, for transmission or storing purposes. Such a system, called a maximally decimated analysis bank, is commonly used in several applications such as speech coding [42], [43], image coding [44], short-term spectral analysis [43], and voice privacy systems [45].

At some subsequent stage, it is eventually necessary to combine the subband signals to recover the original signal $x(n)$ as accurately as possible. This reconstruction is done by the synthesis bank which is a collection of $M$ digital filters $F_{k}(z)$. Fig. 9 shows a complete analysis/synthesis system which is often called the quadrature mirror filter (QMF) bank. The downgoing arrows in Fig. 9 represent decimation by a factor of $M$, whereas the upgoing arrows represent the insertion of $M-1$ zero valued samples between adjacent samples, in order to match up the "sampling rates" of $\hat{x}(n)$ and $x(n)$. Details of operation of the system of Fig. 9 can be found in a number of references [46]-[48], including recent tutorial articles [48], [57]. Suffice it to point out here that $\hat{x}(n)$ is a distorted version of $x(n)$ for several reasons. First, there is aliasing caused by undersampling ( since the filters $H_{k}(z)$ prior to decimation 


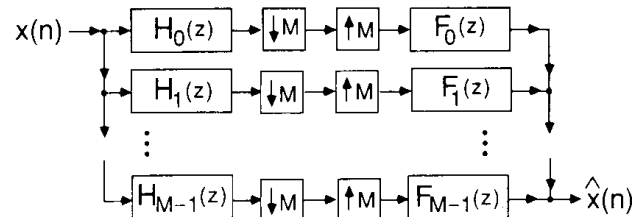

Fig. 9. An $M$ band, maximally decimated parallel QMF bank.

are not ideal bandpass filters). Assuming that aliasing is somehow eliminated, we have a transfer function between $\hat{X}(z)$ and $X(z)$ equal to $T(z)=1 / M \sum_{k=0}^{M-1} H_{k}(z) F_{k}(z)$. This causes amplitude distortion (unless $T(z)$ is forced to be all-pass) and phase distortion (unless $T(z)$ is forced to have linear phase ). If the filters $H_{k}(z)$ and $F_{k}(z)$ are chosen so that $T(z)$ is a delay (i.e., $T(z)=c z^{-n 0}$ ) then $\hat{x}(n)$ is a (delayed) replica of $x(n)$, and the system of Fig. 9 is said to have perfect-reconstruction property.

As such, perfect-reconstruction might seem to be a simple task to accomplish. For example if we take

$$
H_{k}(z)=z^{-k}, \quad F_{k}(z)=z^{-(M-1-k)},
$$

then we have $\hat{x}(n)=x(n-M+1)$. However if we simultaneously insist that the analysis filters should have sharp cutoff and good stopband attenuation, then we have a nontrivial design-problem. This problem has recently been handled [18], [48] by using the idea of lossless transfer matrices. Here we shall very briefly state the basic ideas.

First, any analysis filter can be represented in the form $H_{k}(z)=\sum_{l=0}^{M-1} z^{-l} E_{k l}\left(z^{M}\right)$. This is done simply by classifying the impulse response sequence $h_{k}(n)$ into $M$ subsequences $h_{k}(l+n M)$ for $0 \leq l \leq M-1$ and defining $e_{k l}(n)=h_{k}(l+n M)$. The $z$-transform of $e_{k l}(n)$ is then taken as $E_{k l}(z)$. The functions $E_{k l}(z), 0 \leq l \leq M-1$ are called the polyphase components of $H_{k}(z)$. Once we represent the analysis filters in terms of $E_{k l}(z)$, we can repeat a somewhat similar process for the synthesis filters and obtain a representation $F_{k}(z)=\Sigma_{l=0}^{M-1}$ $z^{-(M-1-l)} R_{l k}\left(z^{M}\right)$. Having done so, Fig. 9 can be redrawn as in Fig. 10, where $\boldsymbol{E}(z)=\left[E_{k l}(z)\right]$ and $\boldsymbol{R}(z)=$ $\left[R_{l k}(z)\right]$ are $M \times M$ matrices (called the polyphase component matrices). It is shown in [18], [48] that if $\boldsymbol{E}(z)$ is a lossless transfer matrix and if $\boldsymbol{R}(z)$ is chosen to be $\tilde{\boldsymbol{E}}(z)$ then perfect reconstruction property is ensured. As a result, our design problem is the following: design the analysis filters $H_{k}(z)$ to have good passband and stopband characteristics under the constraint that the related matrix $\boldsymbol{E}(z)$ is lossless.

If $\boldsymbol{E}(z)$ is IIR and lossless then all the poles of $\boldsymbol{R}(z)=$ $\tilde{E}(z)$ are outside the unit circle resulting in instability. For this application $\boldsymbol{E}(z)$ is therefore restricted to be FIR. We can represent $\boldsymbol{E}(z)$ as a product of the form (85) which ensures losslessness. Our job now is to optimize the components of $\boldsymbol{v}_{k}$ and $\boldsymbol{H}_{0}$ such that the filters $H_{k}(z)$ have good responses. Design examples can be found in [18]-[20], [48]. It should be noticed that when the form (85) is op-

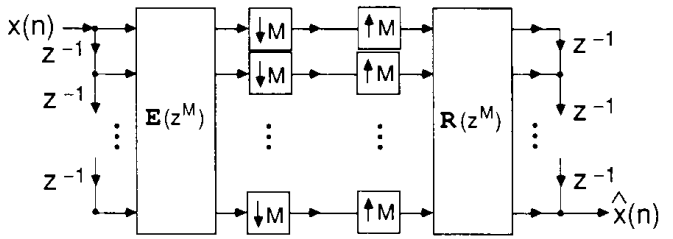

Fig. 10. Redrawing of Fig. 9, in terms of the polyphase component matrices.

timized, the optimization routine searches over every $M$ $\times M$ FIR lossless system with a given degree. In other words the optimization takes place over the complete set of suitable transfer matrices.

\section{Further Remarks and Suggested Reading}

There exists a large body of literature on lossless systems and their applications. In this tutorial we have attempted to cover the basics in a reasonably complete fashion, so that some of the deeper literature in this area can be comprehended and used with relative ease.

Design examples are undoubtedly excellent tools for familiarizing with some of the concepts discussed in this tutorial. For want of space, we have refrained from reproducing many examples. Here is a short list of relevant design examples. Very illuminating examples on wave filter design can be found in [25, ch. 12]. Examples of FIR lossless lattice structures for low sensitivity design can be found in [13]. The use of FIR lossless lattice structures for two-channel QMF design is demonstrated in [19, pp. $88,89]$ and for $M$ channel case in [18, pp. 488, 489] and [58, pp. 697]. Finally examples of IIR low-sensitivity designs based on the structure of Fig. 7 can be found in [12, Section V], and in [40, Section III].

In the world of classical electrical network synthesis, lossless electrical impedances (called reactance functions [2], [3], [6], [7]) are well known. An impedance $Z(s)$ is lossless if the real (i.e., resistive) part of $Z(j \Omega)$ is zero for all $\Omega$. These impedances are the driving-point functions of networks made of lossless elements (such as inductors and capacitors but no resistors). A very simple mapping which translates such an impedance into an allpass function is given by $G(s)=(Z(s)-1) /(Z(s)+$ $1)$. If we perform a bilinear transformation [24] to convert $G(s)$ into a discrete-time system, the result $H(z)$ is the stable all-pass function discussed in Section II. In a similar fashion, we can define multiport lossless electrical networks characterized by lossless impedance matrices, which can be translated into lossless discrete-time systems. The texts by Balabanian [7], Anderson and Vongpanitlerd [5], Belevitch [4], and Newcomb [28] offer excellent treatments at various levels of the details of different aspects in this connection. An earlier work by Potapov [27] describes several fundamental algebraic properties of lossless systems. As mentioned earlier, the text by Belevitch [4], which is perhaps the only one of its kind, offers great insight into the operations of lossless continuous-time systems. 
A well-known class of low-sensitivity electrical filters is the so called doubly terminated lossless two-ports [6][8]. It is possible to imitate the behavior of these by translating the structure into the digital world. Digital filter structures designed in this fashion are called wave filters [9]. An excellent chapter on wave digital filters can be found in the text by Antoniou [25]. It is also known that wave filters have subtle internal losslessness property which enables the designer to suppress limit cycle oscillations [10].

The direct use of losslessness concept in the $z$ domain (without translating a continuous time network) has been done in [11]-[14], [49]. Digital filter structures based on these possess several important properties including those mentioned in Section V-A.

The Gray and Markel lattice structures which were mentioned in Section V-A form a very important class and are related to many diverse engineering topics such as 1) linear prediction [50], 2) wave lattice filters [34], 3) lossless transmission line theory [51] 4) stability test procedures [16], and 5) orthogonal polynomials [37]. Tutorial treatments of some of these aspects can be found in [16], [29]. Reference [30] is a tutorial emphasizing scalar allpass functions and applications.

The QMF problem discussed in Section V-B has been treated in a number of references. An early tutorial on multirate systems is the paper by Crochiere and Rabiner [52]. The text by Crochiere and Rabiner [43] gives an excellent foundation to polyphase structures and filter banks. The magazine article [48] is a recent tutorial on filter banks and the use of lossless matrices in these systems. A good place to read up the properties of unimodular and other polynomial matrices is the text by Gantmacher [55, vol. 1 , ch. VI].

\section{REFERENCES}

[1] O. Brune. "Synthesis of a finite two terminal network whose drivingpoint impedance is a prescribed function of frequency," $J$. Math. Phys. vol. 10, pp. 191-236, Oct. 1931.

[2] E. A. Guillemin, Synthesis of Passive Networks. New York: Wiley, 1957.

[3] M. E. Van Valkenburg, Introduction to Modern Network Synthesis. New York: Wiley, 1964.

14] V. Belevitch, Classical Network Theory. San Francisco, CA, Holden Day. 1968.

[5] B. D. O. Anderson and S. Vongpanitlerd, Network Analysis and Synthesis. Englewood Cliffs. NJ: Prentice Hall, 1973.

[6] G. C. Temes and J. W. LaPatra, Introduction to Circuit Synthesis and Design. New York: McGraw-Hill, 1977.

[7] N. Balabanian and T. A. Bickart, Electrical Network Theory. New York: Wiley, 1969.

[8] H. J. Orchard, "Inductorless filters, " Electron. Lett, vol. 2, pp. 224 225, Sept. 1966.

[9] A. Fettweis, "Digital filter structures related to classical filter networks." in Digital Signal Processing 11 , IEEE ASSP Soc. NY, IEEE Press, 1975, pp. 475-485.

[10] A. Fettweis and K. Meerkotter. "Suppression of parasitic oscillations in wave digital filters," IEEE Trans. Circuits Syst., vol. CAS-22, pp. 239-246, Mar. 1975.

[11] P. P. Vaidyanathan and S. K. Mitra, " Low passband sensitivity digital filters: A generalized viewpoint and synthesis procedures, "Proc. IEEE, vol. 72. pp. 404-423, Apr. 1984.

[12\} P. P. Vaidyanathan, S. K. Mitra, and Y. Neuvo, "A new approach to the realization of low sensitivity IIR digital filters." IEEE Trans.
Acoust., Speech, Signal Processing, vol. ASSP-34, pp. 350-36I, Apr. 1986.

[13] P. P. Vaidyanathan, "Passive cascaded lattice structures for low sensitivity FIR filter design, with applications to filter banks, " IEEE Trans. Circuits Sist. vol. CAS-33.pp. 1045-1065. Nov. 1986.

[14] P. P. Vaidyanathan and V. Liu. "An improved sufficient condition for absence of limit cycles in digital filters." IEEE Trans. Circuits Syst., vol. CAS-34, pp. 319-322, Mar. 1987.

[15] Ph. Delsarte, Y. Genin, and Y. Kamp, "On the role of the Nevanlinna-Pick problem in circuit and system theory. " Iht. J. Circuit Theory Appl., vol. 9. pp. 177-187. 1981.

[16] P. P. Vaidyanathan and S. K. Mitra, "A unified structural interpretation of some well-known stability test procedures for linear systems," Proc. IEEE, vol. 75, pp. 478-497, April 1987

[17] S. K. Mitra, Y. Neuvo, and H. Roivainen, "Variable cutoff frequency digital filters." in Proc. IASTED Int. Symp. Appl. Sig. Proc. Digital Fillering. Paris. France, June 1985.

[18] P. P. Vaidyanathan. "Theory and design of $M$-channel maximally decimated quadrature mirror filters with arbitrary $M$. having perfect reconstruction property, "IEEE Trans. Acoust., Speech, Signal Processing, vol. ASSP-35, pp. 476-492, Apr. 1987

[19] P. P. Vaidyanathan and P.-Q. Hoang, "Lattice structures for optimal design and robust implementation of two-channel perfect reconstruction QMF banks," IEEE Trans. Acoust., Speech. Signal Processing, vol. ASSP-36, pp. 81-94, Jan. 1988.

[20] P. P. Vaidyanathan, Z. Doganata, and T. Q. Nguyen, "More results on the perfect reconstruction problem in $M$-band parallel QMF banks." in Proc. IEEE Intern. Symp. Circuits Sist.. Philadelphia. PA, May 1987, pp. 847-850.

[21] P. P. Vaidyanathan and V. C. Liu. "Sampling theorem in the context of polyphase digital filter banks," in Proc. 21st Annu. Asilomar Conf. Signals, Syst., Comput., Nov. 1987. CA. to be published.

[22] C. A. Desoer and J. D. Schulman, "Zeros and poles of matrix transfer functions and their dynamical interpretation." IEEE Trans. Circuits Syst., vol. CAS-21. pp. 3-8, Jan. 1974.

[23] T. Kailath, Linear Systems. Englewood Cliffs, NJ: Prentice-Hall, 1980.

[24] A. V. Oppenheim and R. W. Schafer, Digital Signal Processing. Englewood Cliffs, NJ: Prentice-Hall, 1975.

[25] A. Antoniou, Digital Filters: Analysis and Design. New York: McGraw-Hill, 1979.

[26] P. P. Vaidyanathan and S. K. Mitra, "A general family of multivariable digital lattice filters," IEEE Trans. Circuits Syst., vol. CAS-32. pp. 1234-1245, Dec. 1985

[27] V. P. Potapov, “The multiplicative structure of J-contractive matrix functions," Amer. Math. Soc.. Translation Series 2, vol. 15, pp. 131243. 1960.

[28] R. W. Newcomb, Linear Multiport Synthesis. New York: McGraw Hill, 1966.

[29] P. P. Vaidyanathan. "The discrete-time bounded real lemma in digital filtering," IEEE Trans. Circuits Syst, vol. CAS-32, pp. 918924, Sept. 1985.

[30] P. A. Regalia, S. K. Mitra, and P. P. Vaidyanathan, "The digital allpass filter: A versatile signal processing building block." Proc. IEEE, pp. 19-37. 1988.

[31] A. G. Constantinides, "Frequency transformations in digital filters," Electron. Lett., vol. 3, pp. 487-489, Nov. 1967

[32] J. Franklin, Matrix Theory. Englewood Cliffs. NJ: Prentice-Hall, 1968.

[33] F. D. Murnaghan. The Unitary and Rotation Groups. Washington. DC: Spartan, 1962

[34] L. Gaszi, "Explicit formulas for lattice wave digital filters," IEEE Trans. Circuits Syst, vol. CAS-32. pp. 68-88. Jan. 1985.

[35] T. Saramaki, "On the design of digital filters as a sum of two allpass filters," IEEE Trans. Circuits Syst., vol. CAS-32, pp. 1191-1193. Nov. 1985

[36] S. K. Mitra and K. Hirano, "Digital allpass networks." IEEE Trans. Circuits Syst., vol. CAS-21. pp. 688-700. Sept. 1974.

[37] A. H. Gray. Jr. and J. D. Markel. "Digital lattice and ladder filter synthesis," IEEE Trans. Audio Electroacoust., vol. AU-21, pp. 491500, Dec. 1973

[38] A. H. Gray, Jr. and J. D. Markel, "A normalized digital filter structure," IEEE Trans. Acoust., Speech, Signal Processing, vol. ASSP23. pp. 268-277. June 1975.

[39] A. H. Gray, Jr., "Passive cascaded lattice digital filters," IEEE Trans. Circuits Syst., vol. CAS-27, pp. 337-344. May 1980.

[40] P. P. Vaidyanathan. P. Regalia, and S. K. Mitra. "Design of doubly 
complementary IIR digital filters using a single complex allpass filter, with multirate applications," IEEE Trans. Circuits Syst., vol. CAS34, pp. 378-389, Apr. 1987.

[41] S. Y. Kung, H. J. Whitehouse, and T. Kailath, Eds., VLSI and Modern Signal Processing. Englewood Cliffs, NJ: Prentice-Hall, 1985.

[42] A. Croisier, D. Esteban. and C. Galand, "Perfect channel splitting by use of interpolation/decimation/tree decomposition techniques, Int. Symp. Info., Circuits Systems, Patras, Greece, 1976.

[43] R. E. Crochiere and L. R. Rabiner, Multirate Digital Signal Processing. Englewood Cliffs, NJ: Prentice-Hall, 1983

[44] J. W. Woods, and S. D. O'Neil, "Subband coding of images," IEEE Trans. Acoust., Speech, Signal Processing, vol. ASSP-34, pp. 12781288, Oct. 1986.

[45] R. V. Cox, D. E. Bock, K. B. Bauer, J. D. Johnston, and J. H. Snyder, "The analog voice privacy systems," in Proc. IEEE Int. Conf. ASSP, Apr. 1986, pp. 341-344.

[46] M. J. T. Smith and T. P. Barnwell, III, "A new filter-bank theory for time-frequency representation," IEEE Trans. Acoust., Speech Signal Processing, vol. ASSP-35. pp. 314-327. Mar. 1987.

147] M. Vetterli, "A theory of multirate filter banks," IEEE Trans. Acoust., Speech, Signal Processing, vol. ASSP-35, pp. 356-372, Mar. 1987

[48] P. P. Vaidyanathan, "Quadrature mirror filter banks, $M$-band extensions and perfect-reconstruction techniques," IEEE ASSP, vol. 4, pp. 4-20, July 1987.

[49] E. Deprettere and P. Dewilde. "Orthogonal cascade realization of real multiport digital filters," Int. J. Circuit Theory Appl., vol. 8, pp. $245-272,1980$

[50] J. Makhoul, "Stable and efficient lattice methods for linear prediction," IEEE Trans. Acoust., Speech, Signal Processing, vol. ASSP25, pp. 423-428, Oct. 1977.

[51] J. D. Markel and A. H. Gray, Jr. Linear Prediction of Speech. New York: Verlag, 1976.

[52] R. E. Crochiere and L. R. Rabiner, "Interpolation and decimation of digital signals: A tutorial review," Proc. IEEE, vol. 69, pp. 300331, Mar. 1981

[53] N. K. Bose, Digital Filters: Theory and Applications. New York: Elsevier, 1985

[54] P. P. Vaidyanathan, "A tutorial on allpass functions and lossless systems, with applications," Lecture notes, Dep. EE. Caltech, Mar. 1987.

[55] F. R. Gantmacher, The Theory of Matrices, Vols. 1, 2. New York: Chelsa, 1959

[56] H. W. Schuessler. "A stability theorem for discrete systems," IEEE Trans. Acoust., Speech, Signal Processing, vol. ASSP-24, pp. 8789, Feb. 1976

[57] P. P. Vaidyanathan, "A tutorial on multirate digital filter banks," in Proc. IEEE Int. Symp. Circuits Syst., Espoo, Finland, June 1988, pp. 2241-2248.

[58] T, Q. Nguyen and P. P. Vaidyanathan, "Maximally decimated perfect-reconstruction FIR filter banks with pairwise mirror-image analysis (and synthesis) frequency responses," IEEE Trans. Acoust., Speech, Signal Processing, vol. ASSP-36, pp. 693-706, May 1988.

[59] V. C. Liu and P. P. Vaidyanathan, "On factorization of 2D digital FIR lossless matrices for 2D QMF bank applications," to be published.

[60] P. P. Vaidyanathan, Z. Doganata, and T. Q. Nguyen. "General synthesis procedures for FIR lossless transfer matrices for perfect-recon struction multirate filter bank applications." IEEE Trans. Acoust. Speech. Signal Proc., vol. ASSP-36, pp. 1561-1574. Oct. 1988.

[61] P. P. Vaidyanathan, T. Q. Nguyen, Z. Doganata, and T. Saramaki. "Improved technique for design of perfect reconstruction FIR QMF banks with lossless polyphase matrices," IEEE Trans. Acoust., Speech, Signal Processing, vol. ASSP-37, July 1989. to be published.

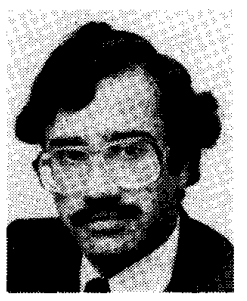

P. P. Vaidyanathan ( $\mathrm{S}^{\prime} 80-\mathrm{M}^{\prime} 83-\mathrm{SM} \mathrm{M}^{\prime} 88$ ) was born in Calcutta, India, on October 16, 1954. He received the B.Sc. (Hons.) degree in physics, and the $B . T e c h$. and $M . T e c h$. degrees in radiophysics and electronics from the University of Calcutta. India, in 1974, 1977, and 1979, respectively, and the Ph.D. degree in electrical and computer engineering from the University of California, Santa Barbara, in 1982

He was a Post-Doctoral fellow at the University of California, Santa Barbara, from September 1982 to February 1983. In March 1983, he joined the California Institute of Technology, Pasadena, as an Assistant Professor of Electrical Engineering, and is currently as Associate Professor in the same Department His main research interests are in digital signal processing, multirate filter bank systems, filter design, adaptive filtering, and multivariable system theory. He served as the Vice-Chairman of the Technical Program Committee for the 1983 IEEE International Symposium on Circuits and Systems and as an Associate Editor for the IEEE Transactions on CiRCuITS AND SYSTEMS, for the years 1985-1987.

Dr. Vaidyanathan was recipient of the award for Excellence in Teaching at the California Institute of Technology, for the year 1983-1984. He is a recipient of NSF's Presidential Young Investigator Award, starting from 1986. In 1988 he received the IEEE ASSP Senior award for his 1987 paper on multirate perfect-reconstruction filter-banks.

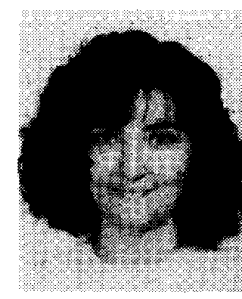

systems.
Zinnur Doğanata ( $\mathrm{S}^{*} 86$ ) was born in Ankara Turkey, on October 29, 1959. She received the B.S. and M.S. degrees in electrical engineering from the Middle East Technical University. Ankara, in 1981 and 1984 , respectively.

During the years 1981-1984, she worked part time for the Military Electronic Industries of Turkey. Presently, she is pursuing the Ph.D. degree in electrical engineering at the California Institute of Technology, Pasadena. Her main research interests are in digital signal processing and lossless 